\title{
Mixing enhancement in coaxial jets through inflow forcing: A numerical study
}

\author{
Guillaume Balarac, ${ }^{\text {a) }}$ Olivier Métais, and Marcel Lesieur \\ Équipe MoST/LEGI B.P. 53, 38041 Grenoble Cedex 09, France
}

(Received 13 September 2006; accepted 15 May 2007; published online 13 July 2007)

\begin{abstract}
Direct numerical simulations are performed to analyze the flow dynamics and the mixing properties of natural (unforced) and excited coaxial jets at moderate Reynolds number. First, the study of the natural coaxial jet, with species injected in the outer jet alone, allows us to understand the role of the coherent vortices on the mixing process during the transition stage. It is observed that the global flow behavior is controlled by the dynamics of the outer shear layer during the transition. The streamwise vortices are shown to play a significant role in the mixing process since they initiate intense ejections from the seeding regions. Spots of pure (unmixed) species from the outer jet are seen to persist far downstream. Two different types of inflow forcing are then considered based on the information provided by the natural coaxial jet: first, a purely axisymmetric excitation and second, combined axisymmetric and azimuthal excitations all of moderate amplitude. These excitations are applied to the outer shear layer with a frequency corresponding to the periodic passage of the outer vortical structures. The goal of these excitations is to trigger the vortices formation and to control their dynamics to improve the mixing properties of the jet. With the purely axisymmetric excitation, the outer and inner Kelvin-Helmholtz vortices appear earlier than for the natural jet and the transition process is faster. This early three-dimensionality growth is due to a rapid appearance of streamwise vortices, stretched between consecutive vortex rings, which lead to enhanced mixing. For the combined axisymmetric and azimuthal excitations, the outer Kelvin-Helmholtz rings appear moreover with an azimuthal deformation from the beginning of the jet. This allows for the early generation of streamwise vortices. The vortex stretching phenomenon takes place near the jet inlet with a growth of the axial component of the vorticity fluctuations. The ejections of species from the outer jet thus appear sooner and with a larger intensity. Finally, the mixing efficiency is studied through the global mixedness and the intensity of segregation. (C) 2007 American Institute of Physics. [DOI: 10.1063/1.2747680]
\end{abstract}

\section{INTRODUCTION}

Coaxial jets are composed of an inner jet surrounded by an annular jet. These are present in various industrial applications and are often used as an effective way of mixing two different fluid streams (chemical engineering systems, combustion devices, ...). The study of the mixing properties of coaxial jets has often focused on the near-field because the largest proportion of the mixing takes place in the developing region containing the potential cores. ${ }^{1}$

As pointed out by Crow and Champagne, ${ }^{2}$ the largescale coherent structures emerging in shear flows play a dominant role in the turbulent transport. Therefore, several authors have focused on the role played by coherent vortices on the near-field mixing in coaxial jets. For instance, Villermaux and $\mathrm{Rehab}^{3}$ showed that the interface between the two streams increases with the instability of the outer shear layer and so the vorticity thickness of the outer shear layer is an important parameter. Ko and co-workers ${ }^{4-6}$ were the first to investigate in detail the near-field of coaxial jets. The coaxial jet presents two shear layers: one between the ambient fluid and the outer jet and another one between the outer jet and the inner jet. They found two sets of vortex rings in the outer

${ }^{a)}$ Electronic mail: guillaume.balarac @ hmg.inpg.fr and inner shear layers and concluded that these vortices exhibit a dynamics similar to the vortex dynamics in a single jet. However, more recent works $^{7-9}$ have shown that the near-field vortex structure of a coaxial jet cannot be considered as a combination of several single jets. Indeed, Dahm et $a .^{7}$ and Wicker and Eaton ${ }^{9}$ found that the vortical motion is dominated by the vortices emerging in the outer shear layer when the annular flow velocity is larger than the central one. For similar velocity ratios, Balarac and Métais ${ }^{10}$ showed that the vortices of the outer shear layer develop with a Strouhal number corresponding to the value predicted by the linear stability theory for the Kelvin-Helmholtz instability of this shear layer. Conversely, they found that the inner vortices are trapped in the free space between two consecutive outer vortices showing that the inner vortices evolution is dictated by the outer vortices motion: it is the "locking" phenomenon. As in plane mixing layers ${ }^{11}$ and single jets, ${ }^{12}$ the coaxial jets develop counter-rotating pairs of streamwise vortices stretched between consecutive vortex rings: these play a major role in the mixing process. Indeed, in a recent numerical study, ${ }^{13}$ we have shown that the ejections of the species seeded in the outer jet associated with these streamwise vortices strongly enhanced the jet mixing properties. Balarac et $a l .{ }^{13}$ also demonstrated that the turbulent mixing process is 
strongly affected by the jet upstream conditions, which modify the transitional state. In particular, inflow initial conditions which favor the appearance of streamwise vortices were seen to strongly enhance the jet mixing properties. For example, Balarac et al. ${ }^{13}$ varied the velocity ratio $r_{u}$ $=U_{2} / U_{1}$ between the outer jet velocity $U_{2}$ and the inner jet velocity $U_{1}$. It is well known ${ }^{14}$ that the jet develops a large reverse flow region, called recirculation bubble, for very high velocity ratio. The recirculation bubble is seen to favor ${ }^{13}$ the production of intense streamwise just downstream of the bubble ${ }^{15}$ and consequently enhance the jet mixing.

The goal of the present work consists of proposing inflow forcing procedures designed to increase the mixing of coaxial jets. Several experimental and numerical studies have been devoted to the influence of inlet conditions for single jets, but it is only recently that forcing procedures have been proposed for coaxial jets. ${ }^{16-18}$ Furthermore, coaxial jets control has nowadays been investigated mainly through experimental means. The dynamics of free shear flows being mainly determined by the development of the coherent structures near the flow inlet, several studies have concentrated on the manipulation of the flow vortices through passive or active control. As far as the single jet is concerned, the main motivations consist of reducing the emitted noise or in enhancing its mixing capabilities. Passive control techniques consist of the modification of the nozzle shape $^{19}$ or in adding "tabs" or "chevrons" on a circular nozzle. ${ }^{20,21}$ Active jet control is obtained through energy consuming devices which create a deterministic perturbation at the jet inlet. In practice, this can be obtained by using loudspeakers, ${ }^{2}$ flap actuators, ${ }^{16}$ or microjets. ${ }^{22}$ Concerning single jets, numerical studies are able to mimic these active control devices by adding a deterministic perturbation at the jet inlet. Thus, da Silva and Métais ${ }^{23}$ mimic the forcing methods used in experimental bifurcating jets (see Reynolds et $a l .^{24}$ for a review). Their studies of the coherent vortex dynamics show that an inlet excitation which combines an axisymmetric excitation at the most amplified unstable frequency and a flapping excitation at the subharmonic frequency allows a spectacular increase of the jet spreading in one preferential direction. They show the possibilities of controlling the jets even at high Reynolds number and with a lower forcing amplitude than the amplitude used in other numerical works. ${ }^{25}$ Other numerical (DNS/LES) works ${ }^{26-28}$ determine the most efficient forcing frequencies but they use also high forcing amplitudes. Several recent numerical works have been devoted to the influence of inlet conditions on the radiated sound by turbulent jets (see Bodony and Lele ${ }^{29}$ for an extensive review). Among them one may quote Morris $e t$ $a l^{30}$ and Bogey and Bailly, ${ }^{31,32}$ who systematically investigated the role of inflow forcing and Andersson et al. ${ }^{33}$ Note that all the previously quoted works were only devoted to single jets.

As far as coaxial jets are concerned, the recent development of microelectromechanical systems (MEMS) makes new ways of control possible. ${ }^{16-18}$ These works show that a nonaxisymmetric forcing (based on microflap actuators) allows a faster development of the streamwise vortices, and it is observed that this forcing leads to mixing enhancement. In these studies, the forcing is applied only on the outer shear layer because of its domination on the jet dynamics. Ritchie et al. ${ }^{34}$ developed an active mixing control through synthetic jets placed circumferentially around the main jet. Thus, large-scale structure modifications, especially in the outer mixing layer, have a large effect on the mixing properties of coaxial jets. Inspired by the previous experimental studies on coaxial jets control, the present work is the first numerical study devoted to the design of deterministic inflow perturbations of low amplitude aimed at controlling the jet mixing. The present study complements our previous works ${ }^{13}$ for which only random inlet perturbations were used with no deterministic forcing. We follow a methodology close to the one adopted by da Silva and Métais ${ }^{23}$ for the single jet. The coaxial jets considered have moderate Reynolds number allowing us to use direct numerical simulations (DNS) (Re $=U_{2} D_{1} / \nu=3000$ where $U_{2}$ is the outer jet velocity and $D_{1}$ is the inner jet diameter).

This paper is organized as follows: The next section details the numerical methods and the flow parameters used in the calculations, and the different types of forcing used in this work are also presented. In Sec. III, the dynamics and mixing properties of the natural (unforced) coaxial jet is briefly recalled. We then investigate the modifications induced by the various types of excitations on the vortex structures and mixing abilities. A quantification of the mixing efficiency based on the global mixedness and on the intensity of segregation is performed. The last section is devoted to some concluding remarks.

\section{NUMERICAL METHOD AND FLOW PARAMETERS}

\section{A. Numerical method}

The same numerical code is used to perform all the computations presented here. The constant density Navier-Stokes equations, written in Cartesian coordinates, are solved in a parallelelipedic computational domain. The spatial discretization uses a mixed pseudospectral scheme (Canuto et al. ${ }^{35}$ ) in the two transverse directions taken as periodic, $y$ and $z$, and a sixth-order-compact scheme $\left(\right.$ Lele $\left.^{36}\right)$ in the streamwise direction, $x$. The time advancement is assured by a third order Runge-Kutta scheme (Williamson ${ }^{37}$ ). Pressure-velocity coupling is modelled by a fractional step method requiring us to solve a Poisson equation to ensure incompressibility of the velocity field. The outlet boundary condition uses a nonreflective outflow condition $\left(\right.$ Orlanski ${ }^{38}$ ) where the convective and viscous terms are explicitly advanced. Gonze ${ }^{39}$ showed that the coherent structures are not distorted by this condition when leaving the computational domain. The mixing is studied by considering the mixture fraction, $f$, of the species seeded within the outer annular jet at the jet inlet. Thus, $f$ $=1$ (respectively, 0) if there are only species seeded in the outer annular at the inlet (respectively, if there are only species seeded in the remainder of the upstream jet: the inner jet and the co-flow). The mixture fraction evolution is given by a transport equation (convection-diffusion) which is solved simultaneously with the Navier-Stokes equations. For the spatial discretization of the convection term, we use a 


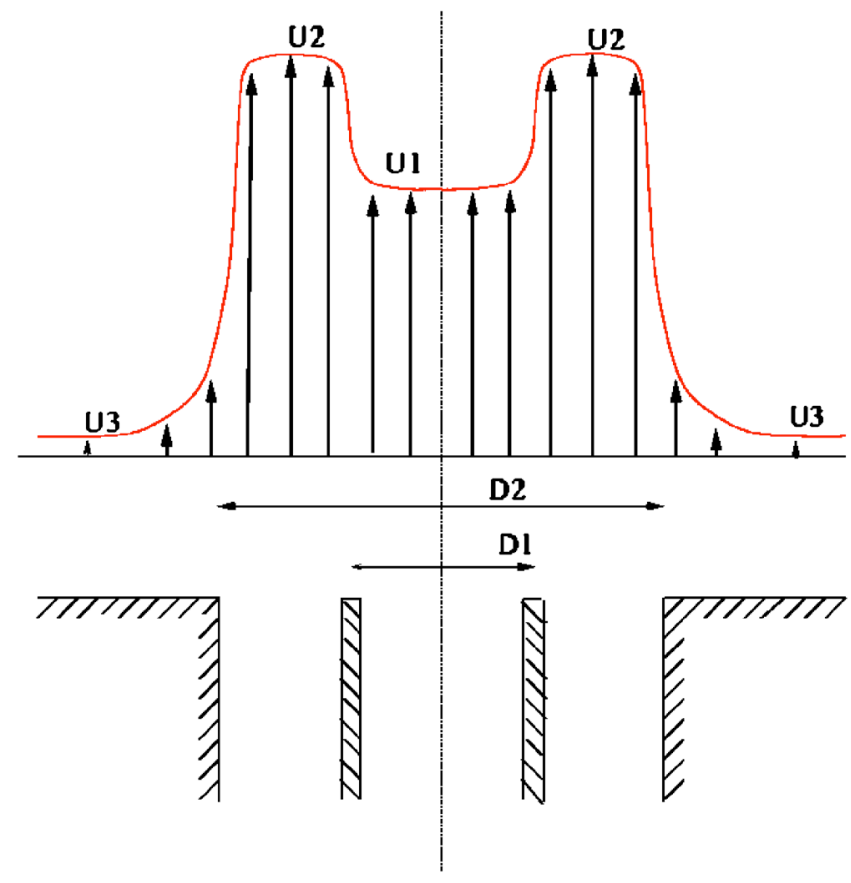

FIG. 1. (Color online) Sketch of the inlet velocity profile. $\theta_{0_{1}}$ and $\theta_{0_{2}}$ are the momentum thicknesses for the inner shear layer (velocity jump from $U_{1}$ to $U_{2}$ ) and for the outer shear layer (velocity jump from $U_{2}$ to $U_{3}$ ), respectively.

second-order semidiscretized TVD Roe scheme. ${ }^{40}$ This code has been previously used in several studies of plane, ${ }^{41}$ round, ${ }^{23}$ and coaxial ${ }^{13,42}$ jets.

The inlet condition is given by a velocity profile in Cartesian coordinates $(x, y, z), \quad \vec{U}\left(\vec{x}_{0}, t\right)=\left(U\left(\vec{x}_{0}, t\right)\right.$, $\left.V\left(\vec{x}_{0}, t\right), W\left(\vec{x}_{0}, t\right)\right)$, prescribed for each time step. Note that we use also the cylindrical coordinates $(x, r, \phi)$ for results analysis. The inlet velocity profile has the shape

$$
\vec{U}\left(\vec{x}_{0}, t\right)=\vec{U}_{\text {coax }}\left(\vec{x}_{0}\right)+\vec{U}_{\text {noise }}\left(\vec{x}_{0}, t\right)+\vec{U}_{\text {forc }}\left(\vec{x}_{0}, t\right),
$$

$\vec{U}_{\text {coax }}\left(\vec{x}_{0}\right)=\left(U_{\text {coax }}\left(\vec{x}_{0}\right), 0,0\right)$ mimics a realistic experimental profile of coaxial jets, $U_{\text {coax }}\left(\vec{x}_{0}\right)$ is constructed by two hyperbolic tangent profiles,

$$
\begin{aligned}
U_{\text {coax }}\left(\vec{x}_{0}=\right. & (0, r, \phi)) \\
& = \begin{cases}\frac{U_{1}+U_{2}}{2}+\frac{U_{1}-U_{2}}{2} \tanh \left(\frac{r-R_{1}}{2 \theta_{01}}\right) & \text { for } r<R_{m} \\
\frac{U_{2}+U_{3}}{2}+\frac{U_{2}-U_{3}}{2} \tanh \left(\frac{r-R_{2}}{2 \theta_{02}}\right) & \text { for } r>R_{m} .\end{cases}
\end{aligned}
$$

In Eq. (2), $U_{1}, U_{2}$, and $U_{3}$ are the inner jet, the outer jet, and the co-flow velocities, respectively. Moreover, $R_{1}, R_{2}$, and $R_{m}=\left(R_{1}+R_{2}\right) / 2$ are the inner, the outer, and the averaged radii, and $\theta_{01}$ and $\theta_{02}$ are the inlet momentum thicknesses of the inner and outer shear layers, defined in terms of $D_{1}$ in Sec. III C below. A sketch of the inlet velocity profile is given by Fig. 1. The inlet mixture fraction profile is also built with hyperbolic tangents (see Balarac et $a l .{ }^{13}$ ). We prescribed

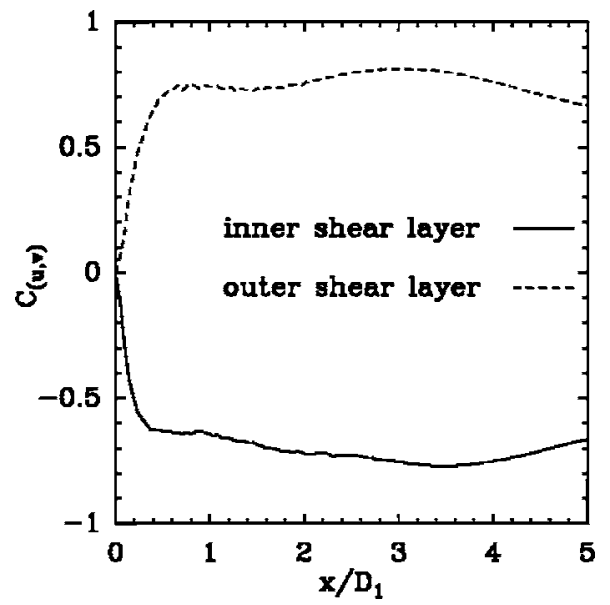

FIG. 2. Downstream evolution of the correlation $C_{(u, v)}$ near the beginning of the jet.

$f=1$ in the outer annular jet and $f=0$ in the remainder of the flow.

$\vec{U}_{\text {noise }}\left(\vec{x}_{0}, t\right)$ is a random noise superimposed on the inlet profile. It is given by

$$
\vec{U}_{\text {noise }}\left(\vec{x}_{0}, t\right)=A U_{\text {base }}\left(\vec{x}_{0}\right) \vec{f}
$$

where $A$ is the maximum amplitude of the incoming noise limited to $A=3 \%$. The noise is mainly located in the inner and outer shear layers by the $U_{\text {base }}\left(\vec{x}_{0}\right)$ function,

$$
U_{\text {base }}\left(\vec{x}_{0}\right)= \begin{cases}0.5 & \text { if } 0.85>r / R_{1} \\ 1.0 & \text { if } 0.85<r / R_{1}<1.15 \\ 1.0 & \text { if } 0.85<r / R_{2}<1.15 \\ 0 & \text { otherwise. }\end{cases}
$$

This function is defined following experimental measurements which show that the velocity fluctuations, at the jet entrance, are mainly located in the shear layer gradients (see, for example, Fig. 2 in Rehab et al. ${ }^{14}$ ). In Eq. (3), $\vec{f}$ is a random noise applied in the three directions and designed to satisfy a given energy spectrum. Note that the noise is imposed on the three velocity components. More details about the inlet mean velocity profile and the noise in coaxial jets can be found in da Silva et al. ${ }^{15}$ Previous works ${ }^{15,42}$ using the same kind of inlet perturbation have shown a good agreement between the numerical and the experimental data.

Note that a random forcing only mimics the turbulent fluctuations encountered at the inlet of the real jets and that the obtainment of realistic inflow conditions would require much more computational efforts with the computation of the inlet nozzle. As pointed out above, the noise is mainly located within the shear layer gradients in agreement with the experimental observations. One of the weaknesses of the random noise remains however the lack of correlation between the various components of the fluctuating velocity field. Figure 2 shows the downstream evolution of the correlation coefficient 


$$
C_{(u, v)}=\frac{\left\langle u_{x}^{\prime} u_{r}^{\prime}\right\rangle}{\sqrt{\left\langle u_{x}^{\prime 2}\right\rangle\left\langle u_{r}^{\prime 2}\right\rangle}},
$$

for a jet disturbed only by this random noise (Sec. III A). Note that the brackets designate a temporal averaging calculated by accumulated data over 6000 instantaneous fields over a time period which allows more than 30 KelvinHelmholtz vortices to leave the computational domain. It can be clearly seen that, despite the synthetic inlet perturbation with zero correlation, the velocity correlations built very fast with a nearly constant level reached around $x / D_{1} \approx 0.4$.

In Eq. (1), $\vec{U}_{\text {forc }}\left(\vec{x}_{0}, t\right)$ corresponds to the deterministic perturbation imposed to excite the jet. The various forcing excitations used in the present work are described in the next section.

\section{B. Forcing types}

The various excitations are designed to force the appearance of the large axisymmetric rings of the outer shear layer and subsequently manipulate their dynamics. As shown in previous works, ${ }^{7,9}$ coherent vortices in high velocity ratio coaxial jets are strongly dominated by the outer vortices in the near-field. Moreover, the outer vortices travel with a frequency $f_{\text {outer }}$ associated with a Strouhal number corresponding to the value predicted by the linear stability theory, $\mathrm{St}$ $=f_{\text {outer }} \theta_{02} / U_{c 2} \approx 0.033$ with $U_{c 2}$ velocity averaged across the outer shear layer. ${ }^{10}$ All the excitations considered in the present work are performed at the preferential frequency $f_{\text {outer }}$ to trigger the formation of the outer rings. The forcing $\vec{U}_{\text {forc }}\left(\vec{x}_{0}, t\right)=\left(U_{\text {forc }}\left(\vec{x}_{0}, t\right), 0,0\right)$ is only applied to the axial velocity. The excitations is localized in the outer shear layer and is associated with a moderate amplitude to minimize the addition of energy at the inlet. Moreover, $U_{\text {forc }}\left(\vec{x}_{0}, t\right)$ is defined to be always positive. This corresponds to a pure blowing without aspiration which is more realistic as far as practical applications are concerned.

The first type of forcing corresponds with a purely axisymmetric excitation, $U_{\text {forc }}\left(\vec{x}_{0}, t\right)$ is thus given by

$$
U_{\text {forc }}\left(\vec{x}_{0}, t\right)=\epsilon U_{\text {loc }}\left(\vec{x}_{0}\right)\left(1 / 2+1 / 2 \sin \left(2 \pi f_{\text {outer }} t\right)\right) \text {, }
$$

where $U_{\text {loc }}\left(\vec{x}_{0}\right)$ is a function designed to localize the excitation only in the outer shear layer,

$$
U_{\text {loc }}\left(\vec{x}_{0}\right)= \begin{cases}1.0 & \text { if } 0.85<r / R_{2}<1.15 \\ 0 & \text { otherwise }\end{cases}
$$

The maximum amplitude is fixed by $\epsilon$ and is taken equal to $8 \%$ of $U_{2}$ for all the computations. Note that this correspond to a relatively low forcing amplitude, ${ }^{23,43}$ since it is interesting to be able to control the jet dynamics with a low level of added energy.

The second type of forcing has the same amplitude as the previous one but now consists of the superposition of an axisymmetric excitation and an azimuthal disturbance characterized by an azimuthal wave number $N$. It is given by

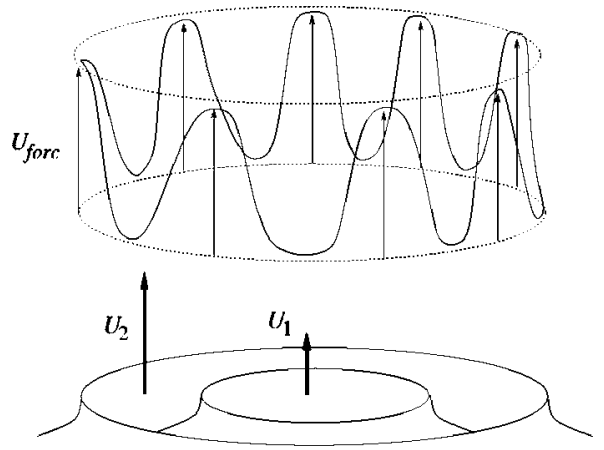

FIG. 3. Sketch of the azimuthal excitation with an azimuthal waves number equal to 8 .

$$
\begin{aligned}
U_{\text {forc }}\left(\vec{x}_{0}, t\right)= & \epsilon U_{\text {loc }}\left(\vec{x}_{0}\right)\left(1 / 2+1 / 2 \sin \left(2 \pi f_{\text {outer }} t\right)\right) \\
& \times(1 / 2+1 / 2 \sin (\phi N)),
\end{aligned}
$$

where $\phi$ is the azimuthal angle of $\vec{x}_{0}$ and $N$ is the azimuthal wave number of the excitation. The role of the azimuthal excitation consists of deforming the axisymmetric rings. A sketch of the azimuthal excitation is given in Fig. 3. The first study concerning the most unstable wave number of an isolated vortex ring was performed by Widnall et al. ${ }^{44}$ Their theory based on inviscid instability gives reasonably good results for very high Reynolds numbers as compared to experimental observation. However, the latter indicates that the number of azimuthal waves increases with the Reynolds number. ${ }^{45}$ Saffman ${ }^{46}$ revisited Widnall's work by including the viscosity effects. This enabled us to predict the dependence of the most unstable wave number with the Reynolds number: a good agreement with the experimental results was obtained. Saffman obtains $N$ as a function of the Reynolds number, the diameter of the nozzle, and the stroke of the piston used to create the isolated vortex ring. In the case presented here, considering the length between two consecutive outer vortex rings instead of the stroke of the piston, it seems that the most unstable wave number is located around $N=10$ [see Eqs. (4.4) and (4.6) in Saffman ${ }^{46}$ ]. To study the influence of $N$, we not only consider the value $N=10$ but also two other values $N=5$ and $N=15$. In fact, this excitation mimics the role played by $N$ microjets placed circumferentially around the outer nozzle and ejecting periodically with a frequency $f_{\text {outer }}$. Microjets have been recently used in laboratory experiments to reduce the single jet noise. Indeed, Arakeri et al. ${ }^{22}$ use microjets ejecting continuously and show the possibility of reducing the noise. In this case, the microjets have similar effects than a co-flow. Pulsed microjets have been used by Choi et al. ${ }^{47}$ and Ibrahim et $a l^{48}$ with different control strategies. These studies show that the microjets have a potential for future use as a device for shear flow control. Numerically, the work of Soteriou et $a .^{49}$ studied the impact of the streamwise vorticity on jet noise by the "vortex method." Their upstream field with 12 equally spaced streamwise vorticity patches distributed around the jet circumference is close to the upstream field given by microjets placed following the radial direction. Finally, let us recall that Ritchie et al. ${ }^{34}$ used nine synthetic jets to control 
coaxial jets and they show the capability of this active control to stimulate the small-scale mixing.

Note that a "vortex ring" forcing procedure was previously designed by Bogey et al. ${ }^{50}$ based on the excitation of a specific combination of the jet azimuthal modes. A random phase was used in order to generate an excitation with a broad frequency range and trigger a rapid generation of small scale turbulence. This is at variance with the present work which uses a deterministic perturbation at the preferential frequency of the jet to favor the formation of the large axisymmetric rings of the outer shear layer. Bogey et al. ${ }^{50}$ furthermore excited a large number of azimuthal modes when we limit our excitation to a given specific azimuthal wave number.

\section{Flow and computational parameters}

All the simulations have been carried out on the same domain. Its size is $10.8 D_{1} \times 10.65 D_{1} \times 10.65 D_{1}$ along the streamwise $(x)$ and the two transverse directions $(y, z)$, and $D_{1}$ is the inner diameter. This domain consists of 231 $\times 384 \times 384$ grid points with uniform mesh size. The inlet velocity profile given by Eq. (2) is defined by the velocity ratio $r_{u}=U_{2} / U_{1}=5$, the radius ratio $\beta=R_{2} / R_{1}=2$, and the initial momentum thicknesses, $D_{1} / \theta_{01}=D_{1} / \theta_{02}=25$. In all the simulations the Reynolds number based on the outer jet velocity and the inner jet diameter is $\operatorname{Re}=U_{2} D_{1} / \nu=3000$. The Schmidt number is taken to be equal to 1 . The various simulations differ only by the nature of the excitation defined by $\vec{U}_{\text {forc }}\left(\vec{x}_{0}, t\right)$. Note that for these values of the parameters, the jet does not exhibit a recirculation region near its inlet (see Balarac and Métais ${ }^{42}$ ).

\section{RESULTS AND DISCUSSIONS}

\section{A. Natural coaxial jets: Flow dynamics and mixing properties}

In this section, we first consider the jet with a white noise perturbation only $\left(\vec{U}_{\text {forc }}\left(\vec{x}_{0}, t\right)=\overrightarrow{0}\right)$. This part briefly summarizes some of the results previously obtained by Balarac et $a l .{ }^{13}$ and constitutes a reference case to which the various forced cases can be properly compared. We will refer to this case with random forcing only as the "natural" jet since there is not a deterministic perturbation added to the inlet profile thus allowing the "natural" development of the instabilities.

Figure 4 shows a global picture of the coherent vortices in the near-field of the natural coaxial jet. It shows isosurfaces of $Q>0$, where $Q$ is the second invariant of the velocity gradient tensor. This positive $Q$ criterion to identify coherent vortices has been originally proposed by Hunt et al. ${ }^{51}$ and has proven to be a good indicator of the coherent vortices (see Dubief and Delcayre ${ }^{52}$ and Lesieur et al $^{53}$ ). The first stage of the transition, until $x / D_{1}=5$, shows the development of inner and outer quasiaxisymmetric vortex rings in both inner and outer shear layers due to Kelvin-Helmholtz instabilities. We can see that the inner Kelvin-Helmholtz vortices are located between two consecutive outer Kelvin-Helmholtz vortices, the inner and outer wavelengths are so equal. This

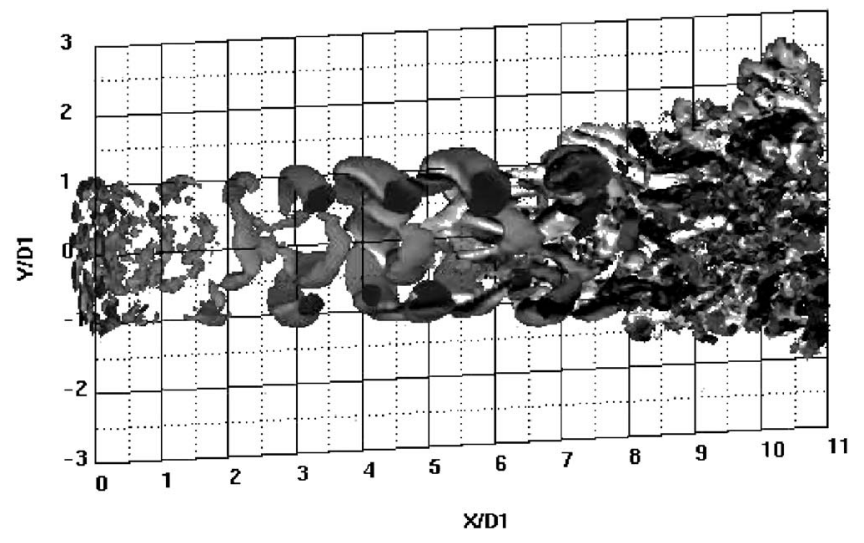

FIG. 4. Visualization of the coherent vortices for the natural (unforced) case by isosurfaces of positive $Q=0.5\left(U_{2} / D_{1}\right)^{2}$ colored by the streamwise vorticity. Light gray corresponds to negative values and dark gray to positive values. Note that a part of the outer rings is artificially cut to show the inner rings.

is due to the "locking" phenomenon mentioned in the Introduction. Around $x / D_{1}=5$, the first streamwise vortices begin to form. They appear by counter-rotating pairs in agreement with the classical scenario of transition in free shear layers. ${ }^{11,12}$ The appearance of longitudinal vortices is accompanied with an abrupt transition towards a developed turbulent state testified by a broad range frequency spectrum with turbulent energy present at all simulated scales. It was furthermore shown that the frequency spectra exhibit a well defined $-5 / 3$ range over about one decade followed by a smooth transition into the dissipative region (see Balarac et al. $\left.{ }^{13}\right)$.

A first view of the mixture fraction evolution is given in Fig. 5 showing the main stages of the mixing process. After a region (until $x / D_{1}=5$ ) where the mixing process is mainly dominated by molecular diffusion, the turbulent mixing develops thanks to coherent vortices. Figure 6 shows that $\langle f\rangle$ remains equal to 0 and 1 in the inner and outer jet, respectively, whereas $\langle f\rangle$ varies at the interfaces (between the inner and outer jet and between the outer jet and the ambient fluid) characterizing the molecular diffusion for $x / D_{1}<5$. At about $x / D_{1}=5$, Kelvin-Helmholtz vortices allow an engulfment of the two streams through the inner and outer shear layers. This implies a radial periodic pulsation due to the ring structures as shown in the animations of Balarac et al. ${ }^{13}$ After $x / D_{1}=6$, views of the mixture fraction contours in transverse sections [Fig. 5(b)] show mushroom-type structures. They characterize the ejection of species seeded in the outer annular jet implied by the counter-rotating streamwise vortices (see also video linked to Fig. 5 online). These ejections allow for the interpenetration of both streams and really initiate the turbulent mixing. Indeed, the rms axial vorticity $\left\langle\omega_{x}^{\prime 2}\right\rangle^{1 / 2}$ exhibits a growth corresponding to the streamwise vortices emergence which is accompanied with a sudden increase of the rms mixture fraction, $\left\langle f^{\prime 2}\right\rangle^{1 / 2}$ (Fig. 7).

Finally, at the end of the transition region, the turbulent scales undergo an intense turbulent mixing. In this region, the mixture fraction profiles (Fig. 6) show that the mean mixture fraction is maximum in the jet center. Thus, large 


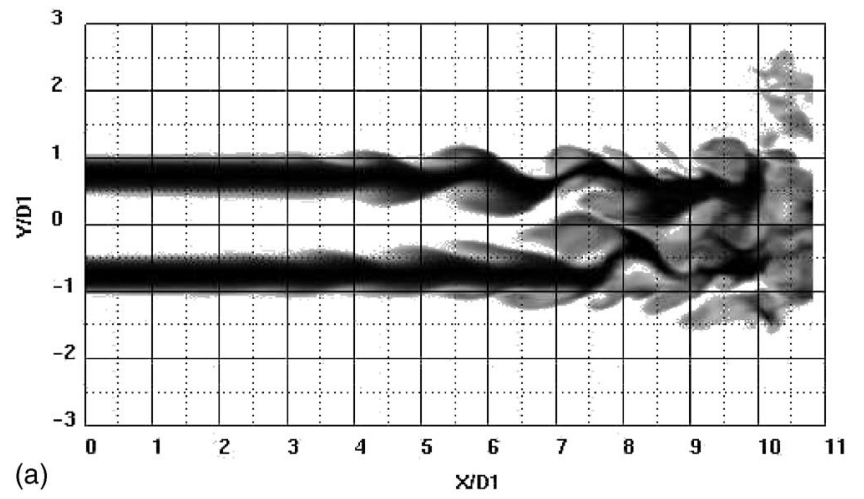

(a)

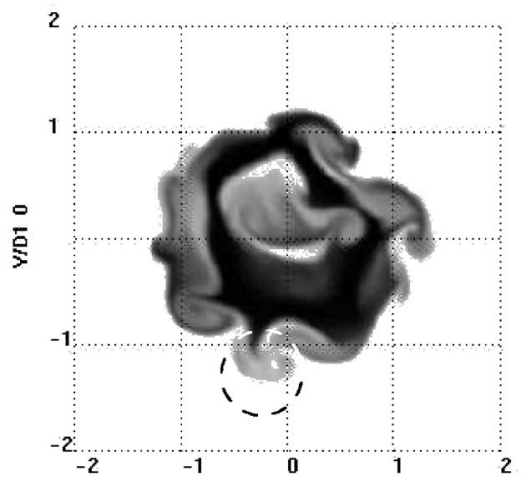

(b)

ZJD1

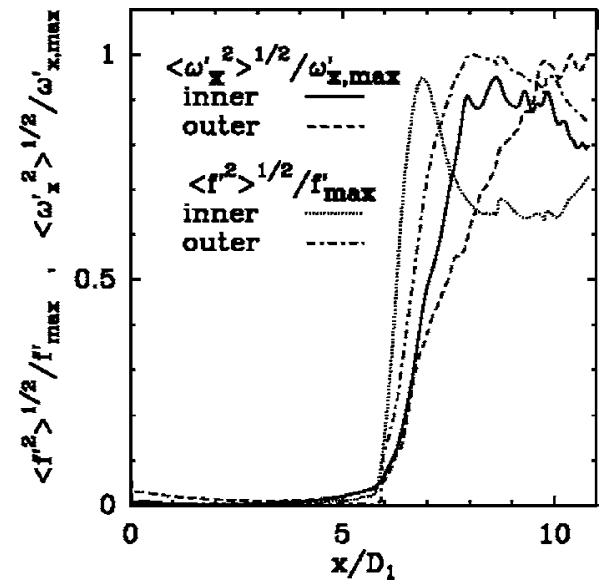

FIG. 7. Downstream evolution of the rms streamwise vorticity and the rms mixture fraction in both inner $\left(r / D_{1}=0\right)$ and outer $\left(r / D_{1}=0.75\right)$ jets in the natural case. Note that each rms quantities is normalized by its maximum rms value $\left(\omega_{x, \max }^{\prime}\right.$ and $f_{\max }^{\prime}$, respectively).

PDF is characterized by a most probable value of $f$ quasiindependent of the radial position and distinct from the mean value, in contrast to a marching PDF where the most probable value equals the mean value. The nonmarching PDF is

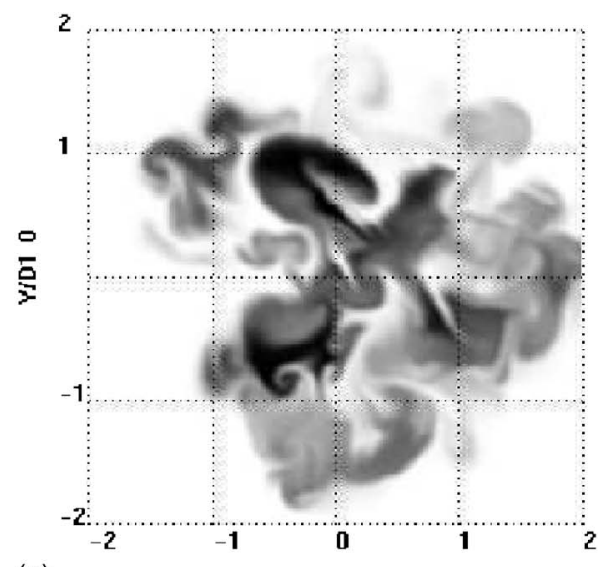

(a) ZD1

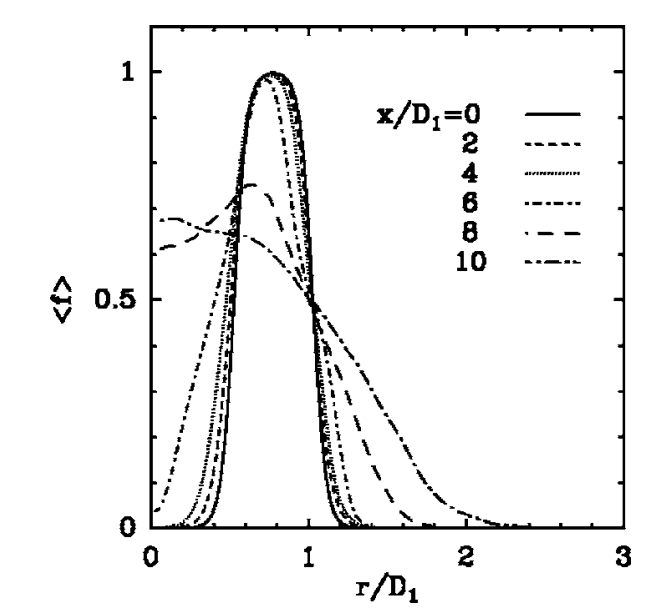

FIG. 6. Mean mixture fraction profiles at several downstream location in the natural case.

FIG. 5. Instantaneous contours of the mixture fraction in the natural case in the central plane (a) and in the transverse section located at $x / D_{1}=6$ (b) (a mushroom-type structure is highlighted by the dashed circle). $f$ varies from 0 (white) to 1 (black) (enhanced online).

amounts of species seeded in the upstream annular jet invade the center of the jet and the inner and outer mixing layers merge in order to create a single mixing zone similar to a single jet. However, Fig. 8(a) shows that regions of unmixed species persist. The study of probability density function (PDF) of the mixture fraction, as performed by Stanley et $a l^{54}$ in plane jets, helps to characterize the mixing in this region. Figure $8(\mathrm{~b})$ shows the mixture fraction PDF at $x / D_{1}=10$ across the only remaining mixing layer. A nonmarching PDF for the mixing layer is found. A nonmarching

Downloaded 26 Jul 2007 to 171.66.39.148. Redistribution subject to AIP license or copyright, see http://pof.aip.org/pof/copyright.jsp 


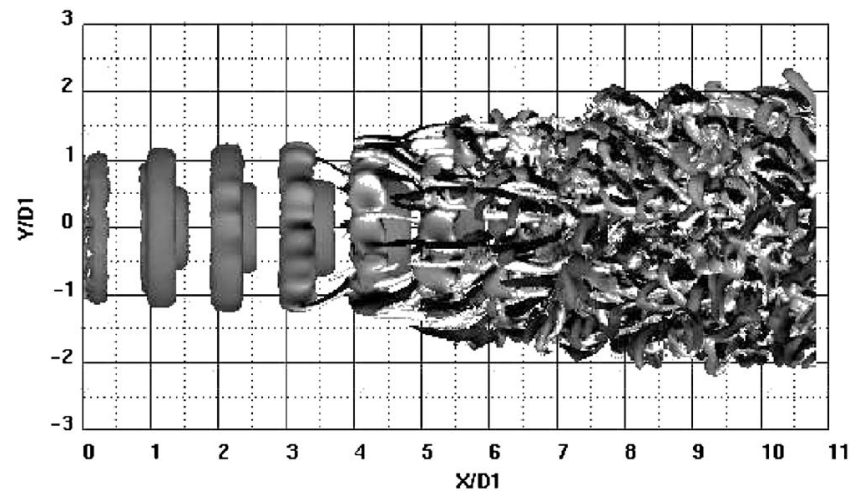

FIG. 9. Visualization of the coherent vortices for a forced (axisymmetric excitation) case. Isosurfaces of positive $Q=0.5\left(U_{2} / D_{1}\right)^{2}$ colored by the streamwise vorticity. Light gray corresponds to negative values and dark gray to positive values.

produced by the persistence of large-scale mixing whereas the marching behavior is the signature of small-scale mixing. ${ }^{55}$ The mixture fraction PDF type shown by Fig. 8(b) reveals an important turbulent mixing activity due to large scales. On the PDF, there are important probabilities to find $f=1$ near the jet center $\left(r / D_{1}=0,0.46\right.$, and 0.92$)$. This constitutes the signature of the remaining large spots of unmixed species. Its reveals that the mixing is not homogeneous in this region. Thus, the goal of the next part is to find appropriate excitations to allow a better homogeneity with a decrease of unmixed species at the end of the computational domain.

\section{B. Axisymmetric excitation}

\section{Flow dynamics}

Here, we investigate the flow dynamics of a coaxial jet under the purely axisymmetric excitation [Eq. (4)]. An instantaneous view of coherent vortices is displayed in Fig. 9 which shows an isosurface of positive $Q$ colored by streamwise vorticity. We can see that the outer Kelvin-Helmholtz rings undergo a rapid amplification and are well formed from the beginning of the jet. Moreover, they exhibit a more axisymmetrical shape than for the unforced case. The inner Kelvin-Helmholtz rings also undergo the influence of the forcing. This is due to the dynamical domination of the outer vortices which impose their motion to the inner structures, which appear earlier and are strongly axisymmetric. The amplification of Kelvin-Helmholtz instabilities allows an acceleration of all the transition stages. Thus, the first streamwise vortices begin to form around $x / D_{1}=3$ between two consecutive outer Kelvin-Helmholtz vortices instead of $x / D_{1}=5$ in the natural case. After $x / D_{1} \approx 4$, the streamwise vortices are well formed and we can observe roughly 15 counter-rotating pairs. Conversely to the outer vortex rings, the inner ones persist during the transition without exhibiting any azimuthal distortion until $x / D_{1} \approx 5$ as shown by Fig. 10 which displays a zoom on an isosurface of $Q>0$ in the inner shear layer (the outer structures have been artificially suppressed). Therefore, if the Kelvin-Helmholtz instability of the outer shear layer influences the formation of the inner Kelvin-Helmholtz vortices from the beginning of the jet, we will see below that it

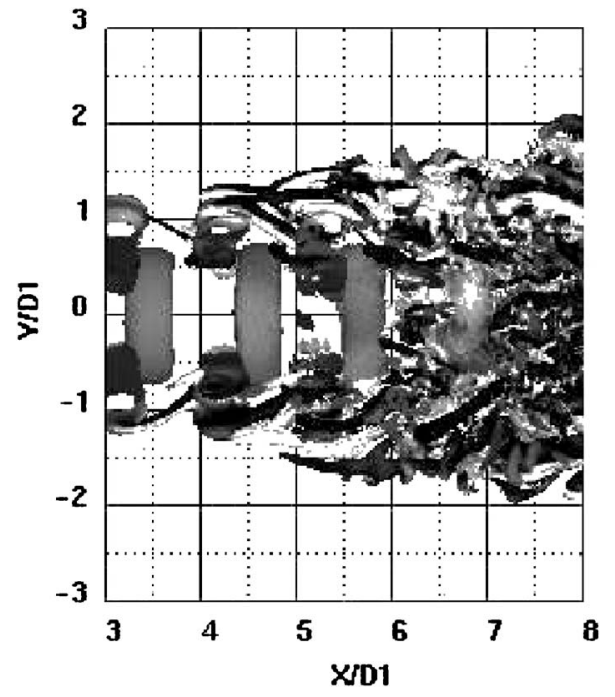

FIG. 10. Zoom on the inner coherent vortices for the axisymmetric excitation. Cut view of positive $Q=0.5\left(U_{2} / D_{1}\right)^{2}$ colored by the streamwise vorticity. Light gray corresponds to negative values and dark gray to positive values.

seems that the azimuthal disturbance of the outer rings during the transition (due to an azimuthal instability) does not influence the inner rings. Finally, the early streamwise vortices appearance allows a rapid growth of the small turbulent structures since the vortex stretching phenomenon linked to the streamwise vortices generation is the dominant factor leading to transition to a fully developed turbulent regime. ${ }^{42}$

A better understanding of the flow modification can be obtained through the turbulent kinetic energy. Thus, we define the following quantities $E_{r}$ and $E_{\phi}$ by ${ }^{15}$

$$
\begin{aligned}
& E_{r}(x)=\sqrt{\left(2 \pi / L_{y} L_{z}\right) \int_{0}^{R_{m}}\left\langle u_{r}^{\prime 2}\right\rangle(x, r) r d r}, \\
& E_{\phi}(x)=\sqrt{\left(2 \pi / L_{y} L_{z}\right) \int_{0}^{R_{m}}\left\langle u_{\phi}^{\prime 2}\right\rangle(x, r) r d r},
\end{aligned}
$$

for the inner shear layer and by

$$
\begin{aligned}
& E_{r}(x)=\sqrt{\left(2 \pi / L_{y} L_{z}\right) \int_{R_{m}}^{\infty}\left\langle u_{r}^{\prime 2}\right\rangle(x, r) r d r}, \\
& E_{\phi}(x)=\sqrt{\left(2 \pi / L_{y} L_{z}\right) \int_{R_{m}}^{\infty}\left\langle u_{\phi}^{\prime 2}\right\rangle(x, r) r d r},
\end{aligned}
$$

for the outer shear layer, respectively. In these equations, $u_{r}^{\prime}$ is the radial component and $u_{\phi}^{\prime}$ is the azimuthal component of the fluctuating velocity. Numerically, Eqs. (8) and (9) are computed by integration from $R_{m}$ to the limit of the numerical domain. These quantities are the contributions of the radial, $E_{r}$, and azimuthal, $E_{\phi}$, Reynolds stresses to the turbulent kinetic energy at a given downstream location, $x$. Thus, $E_{r}$ is directly linked to the development of the jet KelvinHelmholtz instabilities, i.e., with the growth of the vortex rings. Conversely, $E_{\phi}$ is associated with the azimuthal instabilities and constitutes a measure of the three-dimensionality 

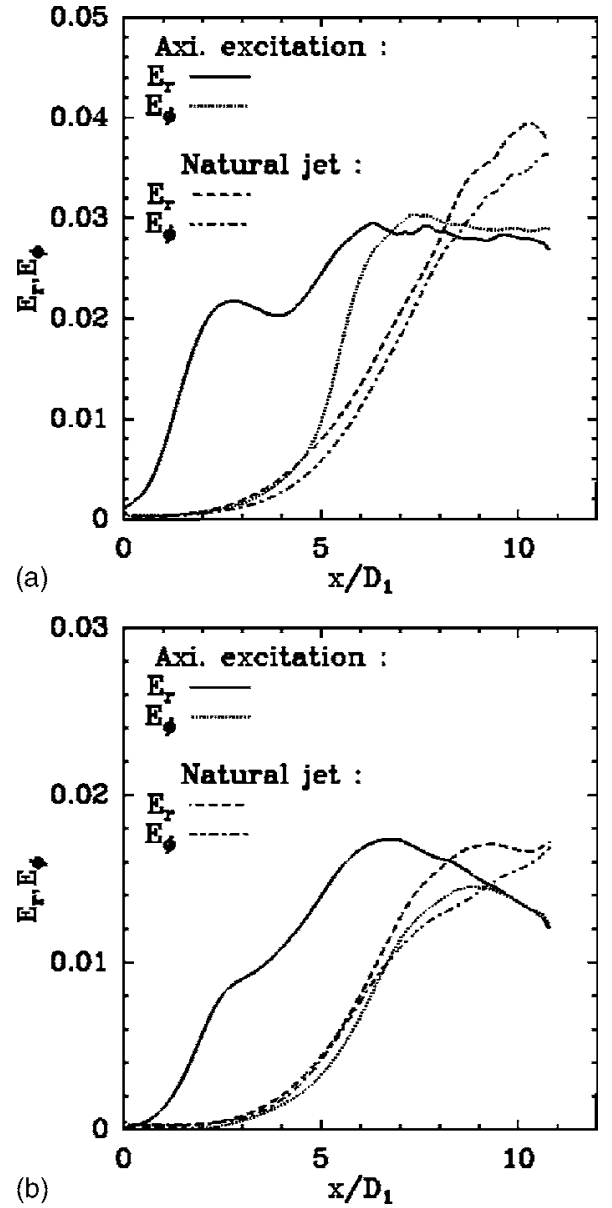

FIG. 11. Downstream evolution of the radial and azimuthal contributions to turbulent kinetic energy calculated in the outer (a) and inner (b) shear layers [see Eqs. (6)-(9) for definition]. Comparison between the axisymmetric excitation and the natural case.

level. For instance, a purely axisymmetric vortex ring (with no swirl) does not contribute to the azimuthal Reynolds stresses component of the turbulent kinetic energy, $E_{\phi}=0$. Figure 11(a) shows the downstream evolution of $E_{r}$ and $E_{\phi}$ for the axisymmetric excitation in the outer shear layer. The same quantities in the natural case are also shown for comparison. Thus, we can see that the forcing implies an earlier growth of these quantities than in the natural case. In the early transition, the main contribution to the turbulent kinetic energy comes from the radial component and $E_{\phi} \approx 0$. This is consistent with the very axisymmetric vortex rings observed in this first region. Moreover, $E_{\phi}$ has a significant contribution from $x / D_{1}=4$ corresponding to the streamwise vortices emergence in the outer shear layer. For the inner shear layer [Fig. 11(b)], the radial contribution is also the main contribution to the turbulent kinetic energy near the entrance of the jet. However, we see that $E_{\phi}$ keeps a behavior very close to the natural case during the transition. Thus, the azimuthal disturbance of the outer shear layer does not seem to influence the inner shear layer in the first steps of the transition (conversely to the Kelvin-Helmholtz instabilities). It is consistent with the axisymmetric persistence of the inner vortices detected by the instantaneous visualizations. Finally, we note for the two shear layers that, in the axisymmetric exci-
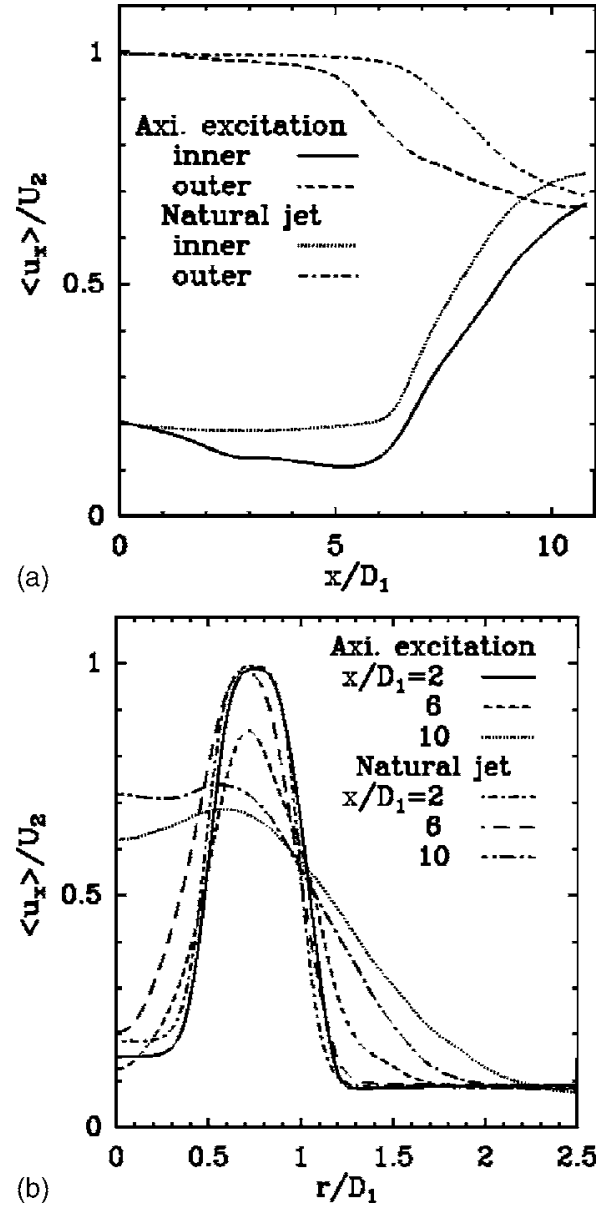

FIG. 12. Comparison of the mean velocity between the axisymmetric excitation and natural cases: (a) downstream evolution in the inner $\left(r / D_{1}=0\right)$ and outer $\left(r / D_{1}=0.75\right)$ jets and (b) profiles at several downstream locations.

tation case, $E_{\phi}$ catches up with $E_{r}$ after $x / D_{1}=7$, showing a full three-dimensionalization of the jet as opposed to the natural case.

The modifications of the transition due to the forcing have a great influence on the mean flow. Thus, there is a diminution of the outer and inner potential core lengths [Fig. 12(a)]. The diminution of the inner potential core length is accompanied with an important decrease of the mean axial velocity along the jet centerline. This is due to the intense inner Kelvin-Helmholtz vortices which entrain an important part of the fluid issued from the inner jet. Moreover, the outer shear layer thickness grows more rapidly in the forced case as shown by the mean velocity profiles at several downstream locations [Fig. 12(b)]. Indeed, at $x / D_{1}=6$, the mean axial velocity profile is close to the initial profile in the unforced case (with a maximum value close to $U_{2}$ ), whereas the mean axial velocity profile at the same downstream location shows an outer shear layer broader in the forced case (with a maximum value of the axial velocity of the order of $0.85 U_{2}$ ). It is because the momentum transfer from the outer annular jet towards the ambient fluid is more pronounced in the forced case due to the early streamwise vortices emergence. Thus, at the end of the computational domain $\left(x / D_{1}=10\right)$, the mean axial velocity profile is broader; $\left\langle u_{x}\right\rangle$ reaches the co-flow value for $r / D_{1} \gtrsim 2.3$ in the forced case instead of 


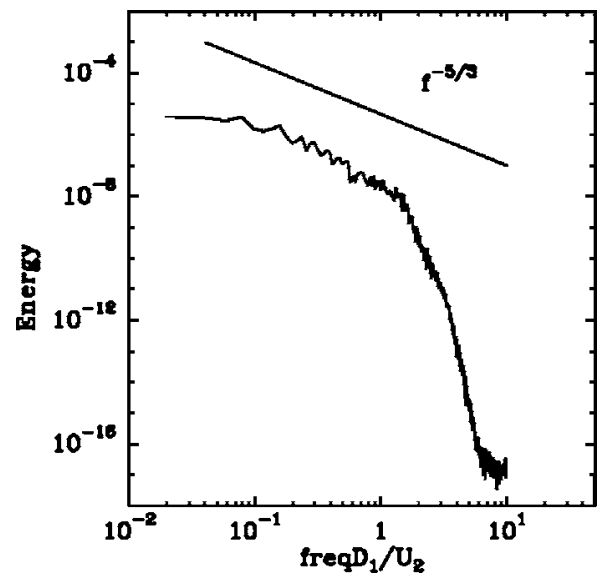

FIG. 13. Frequency spectrum of the axial velocity signal at $x / D_{1}=10$ and $r / D_{1}=0$.

$r / D_{1} \gtrsim 2$ in the natural case. However, at the end of the computational domain, the velocity in the jet center is weaker in the forced case than in the natural case and the maximum value of the mean velocity is not yet located on the center of the jet in the forced case. It is because there is an important decrease of the mean axial velocity along the jet centerline close to the entrance of the jet in the forced case. Thus, the momentum transfer from the outer jet to the inner jet which allows the velocity increase in the center during the transition is not sufficient to homogenize the velocity, conversely to the unforced case. However, in this region, the jet has reached a developed turbulent state as shown by the time spectrum computed from the axial velocity component at $x / D_{1}=10$ (Fig. 13).

\section{Mixing behavior}

Figure 14 shows contours of the mixture fraction in the central plane to illustrate the basic mixing features in the axisymmetric forcing case (see also video linked to Fig. 14 online). We can see that the molecular diffusion stage is shorter in this case as compared with the unforced case (see Fig. 5). This is due to the Kelvin-Helmholtz rings which activate the engulfment of the outer stream as early as $x / D_{1} \approx 1.5$. After $x / D_{1} \approx 4.5$, this stage is followed by the

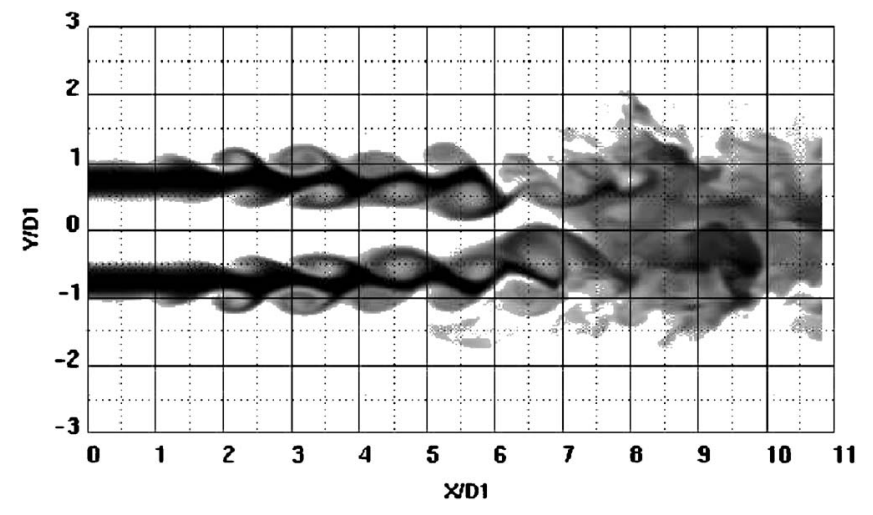

FIG. 14. Instantaneous contours of the mixture fraction for the axisymmetric excitation case in the central plane. $f$ varies from 0 (white) to 1 (black) (enhanced online).
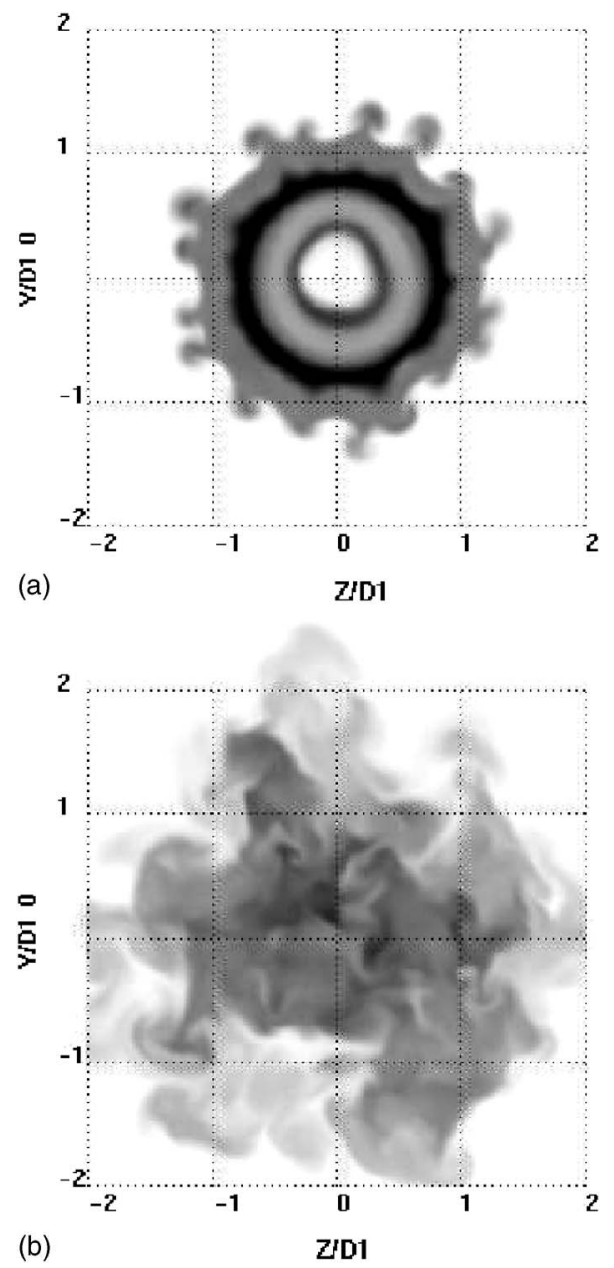

FIG. 15. Instantaneous contours of the mixture fraction in transverse sections for the axisymmetric excitation case. $f$ varies from 0 (white) to 1 (black). (a) $x / D_{1}=4.5$ and (b) $x / D_{1}=10$.

ejection phenomenon associated with the intense streamwise vortices and marked by the appearance of mushroom-type structures [Fig. 15(a)]. It is important to note that no spots of unmixed fluid are visible in the jet core at the end of the computational domain [Fig. 15(b)].

These observations are corroborated by the statistics. Figure 16(a) shows the mean mixture fraction profiles at several downstream locations. The mean mixture fraction profile at $x / D_{1}=4$ confirms that Kelvin-Helmholtz vortices allow a large diffusion of the species issued from the outer jet both in the inner jet and in the ambient fluid. Moreover, at $x / D_{1}=6$, the influence of the outer streamwise vortices ejections is clearly visible through an important widening of the mean mixture fraction profile towards the ambient fluid. However, we can see that the part of the outer species which invades the inner jet is less important than the part of the outer species which invades the ambient fluid. This is because there is no early streamwise vortices generation on the inner shear layer conversely to the outer shear layer to help the mixing between the inner and outer streams as shown during the flow dynamic study (the mixing is only due to the KelvinHelmholtz vortices). It is confirmed by the rms mixture fraction evolution in the downstream direction [Fig. 16(b)]. In- 

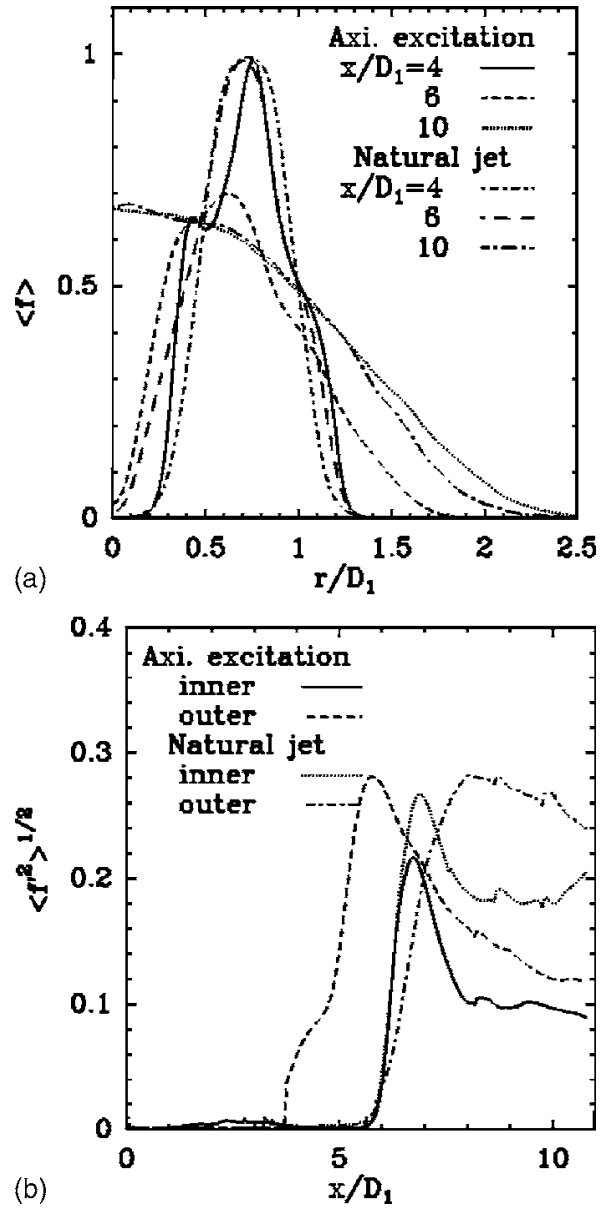

FIG. 16. Mixture fraction statistics comparison between the axisymmetric excitation and the natural case: (a) mean mixture fraction profiles at several downstream locations and (b) downstream evolution of the rms mixture fraction in the inner $\left(r / D_{1}=0\right)$ and outer $\left(r / D_{1}=0.75\right)$ jets.

deed, in the outer jet, $\left\langle f^{\prime 2}\right\rangle^{1 / 2}$ begins to grow earlier in the forced case (at $x / D_{1}=4$ instead of 6) implying a faster turbulent mixing in this region. Whereas in the inner jet, the rms mixture fraction grows around $x / D_{1}=6$ in both cases: forced and unforced. Note that after $x / D_{1}=8$, the rms quantities are smaller in the forced case, showing a diminution of the turbulent mixing activity linked to a better species homogenization.

Figure 17 shows the mixture fraction PDF of the shear layer at $x / D_{1}=10$. Contrary to the natural case, a marchingtype PDF is obtained since the most probable value of $f$ corresponds to the mean value at each radial location. This indicates a homogeneous mixing in this region. Moreover, the probability to find spots of unmixed species $(f=1)$ is zero contrary to the natural case. This indicates that the axisymmetric forcing leads to an enhanced mixing.

\section{Azimuthal excitations}

\section{Flow dynamics}

We now investigate the flow dynamics of a coaxial jet under azimuthal excitations [Eq. (5)]. Instantaneous views of the coherent vortices are shown in Fig. 18 for the three different azimuthal wave numbers. Similarly to the purely axi-

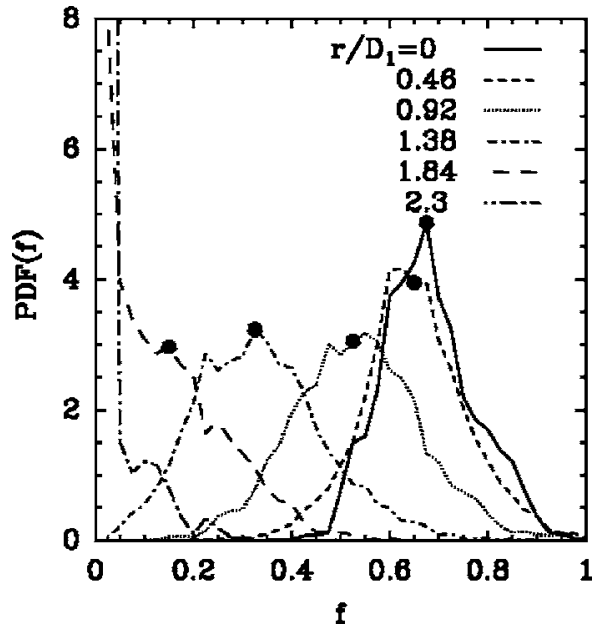

FIG. 17. Variation of the mixture fraction PDF across the mixing layer at $x / D_{1}=10$ in the axisymmetric excitation case, the points show the mean value of $f$ for each radial location.

symmetric forcing, the outer rings quickly form. This is due to the temporal periodic part of the forcing at the preferential mode $f_{\text {outer }}$. As opposed to the purely axisymmetric forcing, the outer rings are no longer axisymmetric due to the azimuthal excitations leading to a deformation of these tori. Figure 19 shows the first outer Kelvin-Helmholtz ring generated by each excitation (purely axisymmetric and combined). We can already see these rings distorted in the azimuthal forced cases conversely to the purely axisymmetric case showing the influence of the azimuthal part from the beginning of the jet. Figure 20 focuses on the inner rings. Conversely to the outer ones, they remain very axisymmetric without azimuthal deformation. This confirms the previous observation that, during the first transition stages, and although the outer shear layer controls the generation of inner Kelvin-Helmholtz instability, it does not seem to influence the inner rings as far as azimuthal instabilities are concerned. The outer Kelvin-Helmholtz vortices distortion is amplified and is quickly followed by the appearance of $N$ counterrotating pairs of streamwise vortices. Moreover, Fig. 18 shows that the outer Kelvin-Helmholtz vortices undergo a more intense azimuthal deformation when the azimuthal wave number is low. Thus, streamwise vortices appear slightly earlier for a low wave number excitation than for a large one or for the axisymmetric forcing case: they are well formed at $x / D_{1} \approx 4$ for $N=15$ and for the axisymmetric forcing case (Fig. 9) instead of $x / D_{1} \approx 3$ for $N=5$ and $N=10$. The azimuthal disturbance associated with the low wave numbers seems to be more quickly amplified. After the streamwise vortices emergence, a lot of small turbulent structures are present and the flow becomes turbulent in all the forced cases.

Figure 21 shows the contribution of the radial and azimuthal Reynolds stresses to the turbulent kinetic energy in the outer and inner shear layers [Eqs. (6)-(9)]. The evolution of these quantities in the outer shear layer is given by Fig. 21(a). Similarly to the axisymmetric forcing case (Fig. 11), both quantities grow earlier than in the natural case, but the azimuthal excitations induce an early growth of $E_{\phi}$. This is 


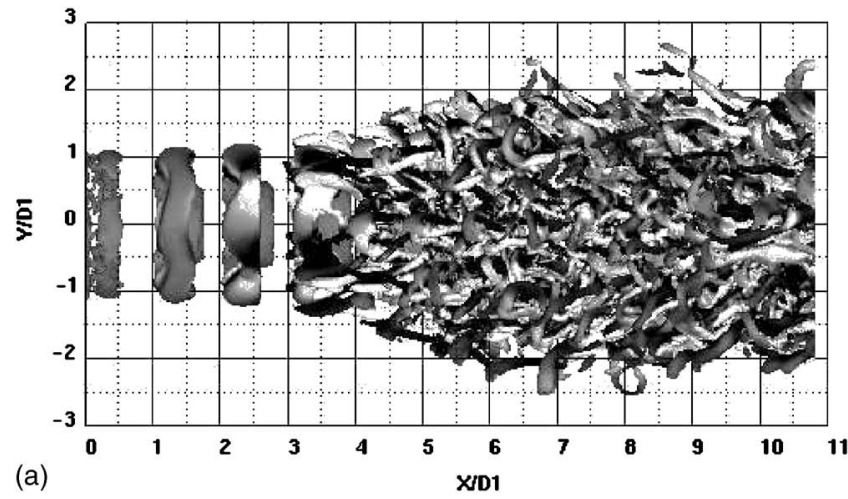

(a)
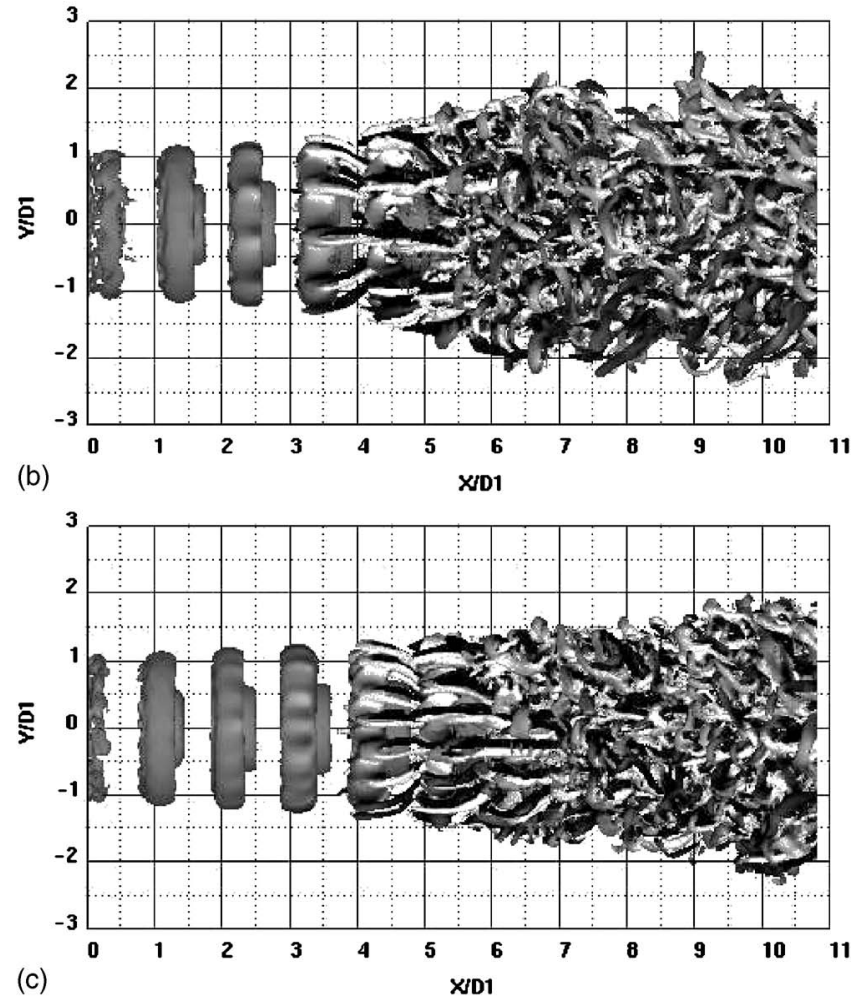

FIG. 18. Visualization of the coherent vortices for forced (azimuthal excitations) cases. Isosurfaces of positive $Q=0.5\left(U_{2} / D_{1}\right)^{2}$ colored by the streamwise vorticity. Light gray corresponds to negative values and dark gray to positive values. (a) $N=5$, (b) $N=10$, and (c) $N=15$.

linked the azimuthal deformation of the outer KelvinHelmholtz rings as soon as they appear. If $E_{r}$ seems independent of $N$, note that the growth of $E_{\phi}$ starts sooner when the azimuthal wave number is low. This is confirmed by Fig. 22, showing the maximum value of the rms streamwise vorticity $\left\langle\omega_{x}^{\prime 2}\right\rangle^{/ 2}$, which is found to be located in the outer shear layer. Thus, the vortex stretching phenomenon leading to the streamwise vortices begins earlier for a small value of $N$ as seen in the flow visualizations. Figure 21(b) shows the same quantities computed for the inner shear layer. We can see that $E_{r}$ dominates $E_{\phi}$ until $x / D_{1} \approx 6$ for $N=15$ instead of $x / D_{1}$ $\approx 5$ for $N=5$. This shows an axisymmetric persistence of the inner Kelvin-Helmholtz vortices for high wave numbers. This axisymmetric persistence is due to the fact that outer Kelvin-Helmholtz rings intensify the axisymmetric part of the inner ones much longer since the azimuthal deformation appears later on the outer rings for large wave numbers. We
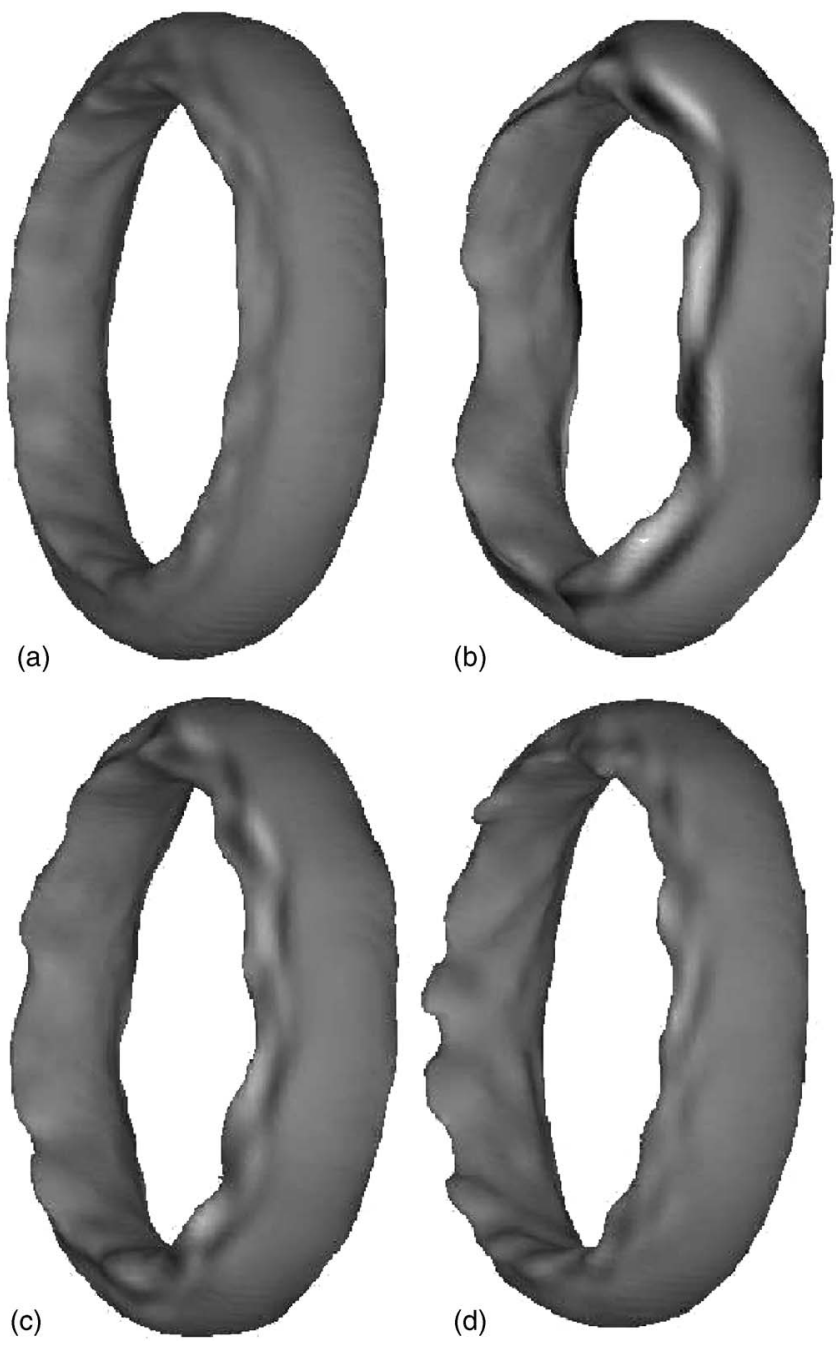

FIG. 19. Zoom on the first outer Kelvin-Helmholtz ring generated by each excitation and shown by an isosurface of positive $Q=0.5\left(U_{2} / D_{1}\right)^{2}$. (a) axisymmetric excitation, (b) $N=5$, (c) $N=10$, and (d) $N=15$.

can note that $E_{r} \approx E_{\phi}$ at the end of the computational domain for the three azimuthal forcing cases, showing a full threedimensionalization similarly to the axisymmetric forcing. As the natural jet and the forced jet with axisymmetric excitation, all the azimuthal forcing cases display a broad range frequency spectrum with a $-5 / 3$ range over about one decade at $x / D_{1}=10$ (Fig. 23). This indicates that the flow exhibits a developed turbulent state at the end of the computational domain.

The azimuthal excitations induce important modifications for the mean flow. As observed in the axisymmetric excitation case, Fig. 24(a) shows a diminution of the outer and inner potential core length with azimuthal excitations. Note that the outer jet velocity decreases earlier for $N=5$ than for $N=15$ because streamwise vortices appear slightly earlier in this case. The decrease of the inner potential core length is accompanied with a diminution of the velocity along the centerline. This is due to the strong entrainment implied by the intense inner Kelvin-Helmholtz vortices. It is interesting to note that the mean axial velocity along the centerline grows earlier for $N=5$ than for $N=15$. To explain that, we recall that there is a "competition" between two 

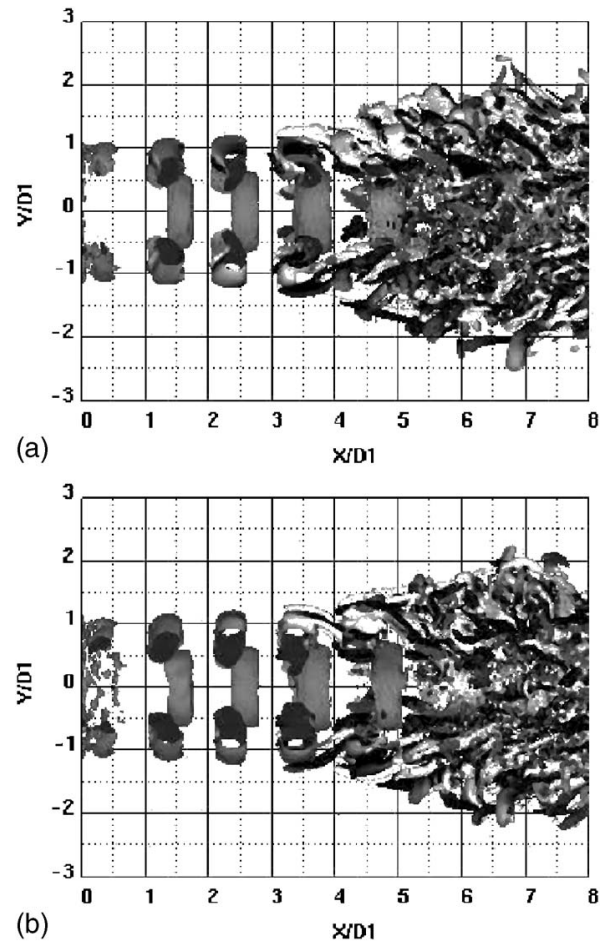

FIG. 20. Zoom on the inner coherent structures for the azimuthal excitation. Cut view of positive $Q=0.5\left(U_{2} / D_{1}\right)^{2}$ colored by the streamwise vorticity. Light gray corresponds to negative values and dark gray to positive values. (a) $N=5$ and (b) $N=10$.

phenomena with antagonistic consequences in this region. First, the entrainment due to the inner Kelvin-Helmholtz vortices inducing a diminution of the axial velocity on the centerline. Second, the momentum transfer phenomenon between the outer and the inner jet due to the vortex stretching phenomenon in the inner shear layer leading to streamwise vortices appearance between two consecutive inner rings. This phenomenon implies an homogenization of the inner and outer jet velocities with the increase of the inner jet velocity. Thus, in the case $N=15$, the azimuthal disturbance appears later and the outer Kelvin-Helmholtz vortices intensify the axisymmetric part of the inner ones as seen before with the contributions of the radial and azimuthal Reynolds stresses to the turbulent kinetic energy in the inner shear layer [Fig. 21(b)]. The natural formation of the streamwise vortices on the inner shear layer is therefore limited and the consequence is an important diminution of the inner jet velocity [Fig. 24(b)]. However, in the case $N=5$, the outer Kelvin-Helmholtz vortices are disturbed earlier and so the streamwise vortices formation on the inner shear layer is not limited allowing the momentum transfer between the inner and outer jets. Figure 25 shows indeed that the streamwise vortices appear earlier on the inner shear layer for $N=5$. The consequence is the growth of the inner jet velocity and the velocity peak shift towards the center as shown by the velocity profile at $x / D_{1}=6$ [Fig. 24(b)]. Finally, the velocity profiles for the different azimuthal excitations are similar at $x / D_{1}=10$. They are wider than the natural case [until $r / D_{1}$ $\approx 2.3$ instead of $r / D_{1} \approx 2$, Fig. 24(b)] due to the $h$ transfer towards the outer shear layer which is intensified by the forcing.
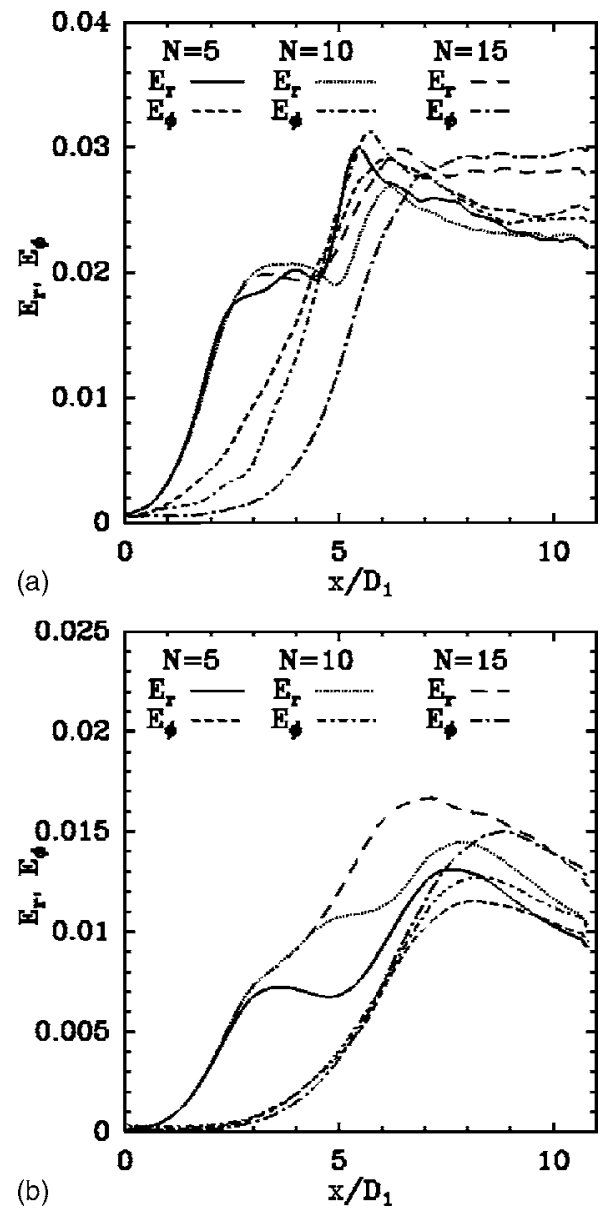

FIG. 21. Downstream evolution of the radial and azimuthal contributions to turbulent kinetic energy calculated in the outer (a) and inner (b) shear layers [see Eqs. (6)-(9) for definition]. Three different azimuthal modes are displayed.

\section{Mixing behavior}

The forcing at the preferential frequency $f_{\text {outer }}$ for both excitation types (purely axisymmetric and combined axisymmetric and azimuthal) leads to some similarities for the mixing behavior (see video linked to Fig. 26 online). Thus, simi-

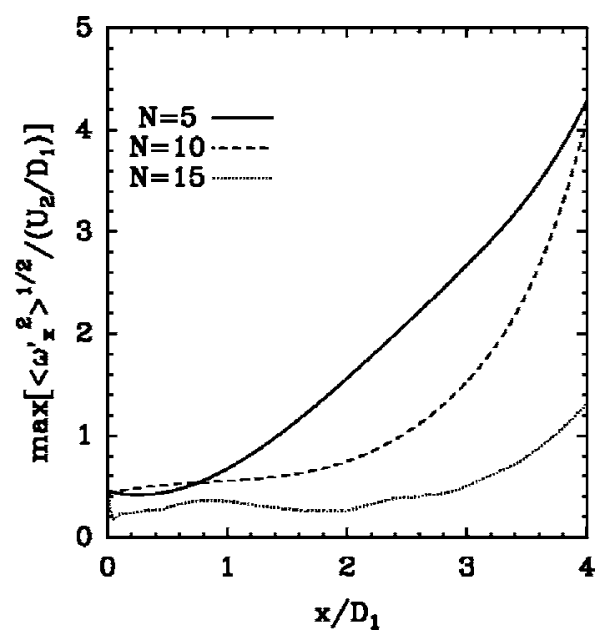

FIG. 22. Downstream evolution of the maximum value of streamwise vorticity rms, $\left\langle\omega_{x}^{\prime 2}\right\rangle^{1 / 2}$, for the three different azimuthal modes. 


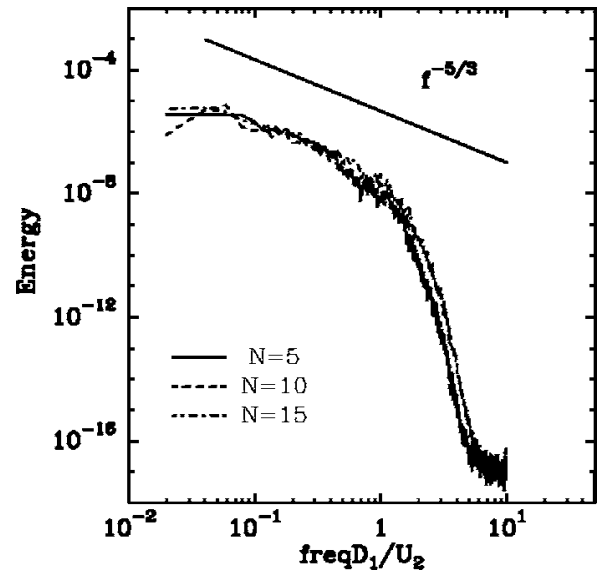

FIG. 23. Frequency spectrum of the axial velocity signal at $x / D_{1}=10$ and $r / D_{1}=0$.

larly to the axisymmetric excitation, the molecular diffusion stage is shorter as compared with the unforced case. This is because Kelvin-Helmholtz vortices are intensified by the forcing and the engulfment of the species seeded in the outer jet starts earlier. However, the instantaneous contours of the mixture fraction in the transverse section (Fig. 26) show significant differences with the purely axisymmetric forcing. In-
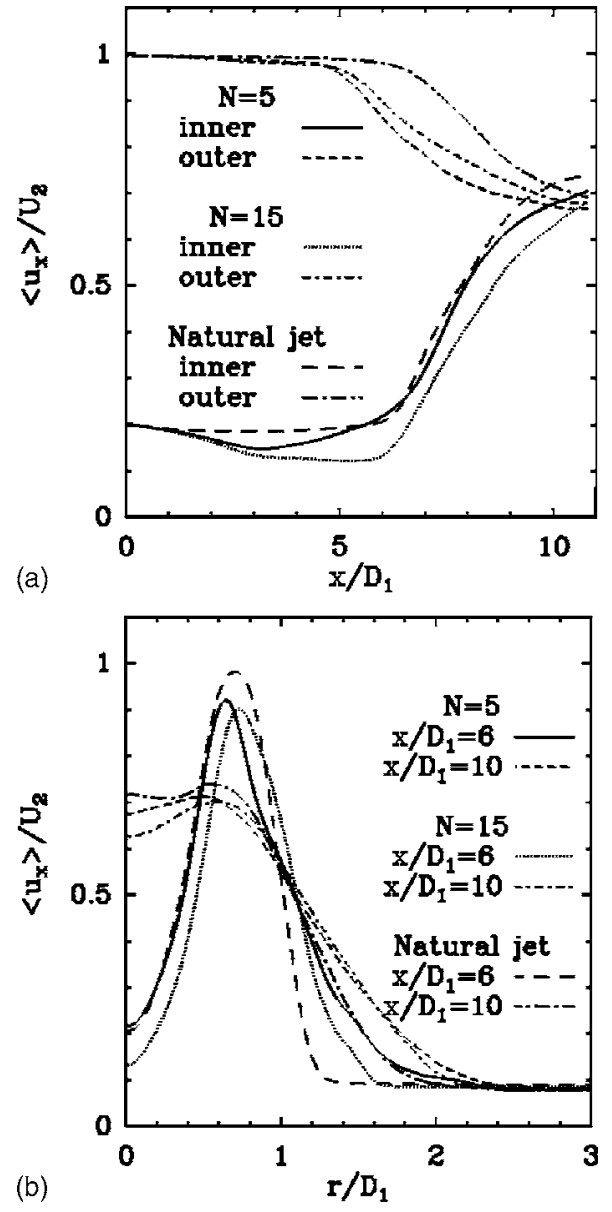

FIG. 24. Comparison of the mean velocity between the azimuthal excitations and natural cases: (a) downstream evolution in the inner $\left(r / D_{1}=0\right)$ and outer $\left(r / D_{1}=0.75\right)$ jets and (b) profiles at several downstream locations.

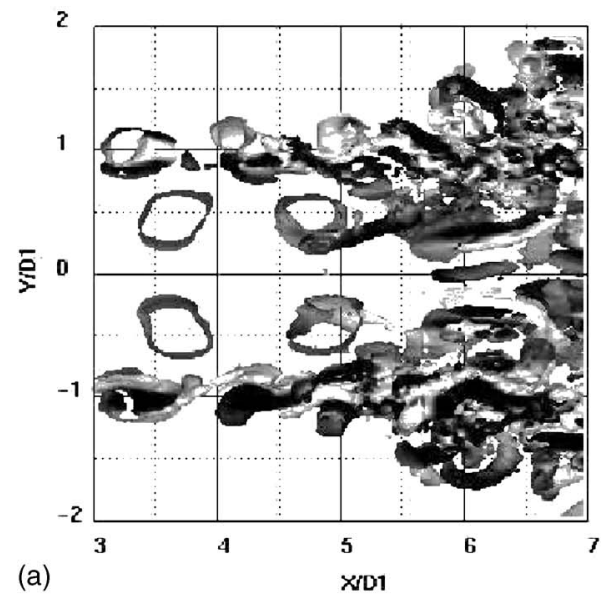

(a)

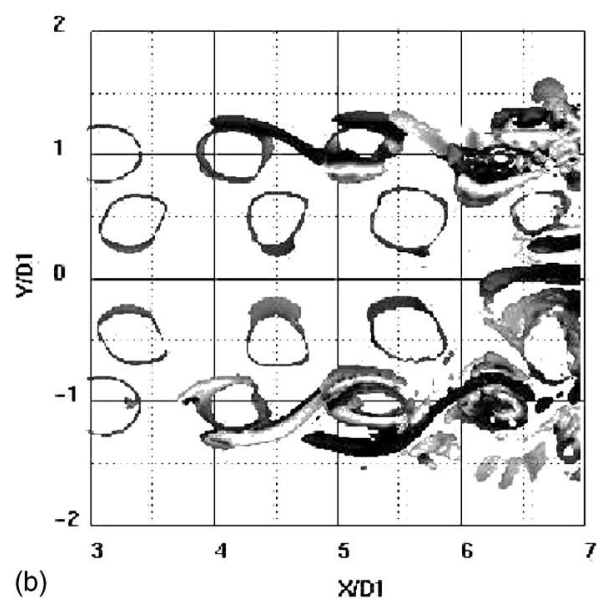

FIG. 25. Illustration of the streamwise vortices appearance in the inner shear layer by isosurfaces of $Q=0.5\left(U_{2} / D_{1}\right)^{2}$. Large parts of the isosurfaces have been cut to see inside the flow. (a) $N=5$ and (b) $N=15$.

deed, the number of ejections, characterized by the mushroom-type structures, is equal to the azimuthal forcing wave number. Moreover, these structures are better organized than for the axisymmetric forcing due to the imposed azimuthal wavelength. It is interesting to note that the intensity of the ejection as measured by the size of a mushroomtype structure increases with decreasing wave numbers. Indeed, this size is roughly $0.7 D_{1}$ for $N=5$ whereas it is roughly $0.4 D_{1}$ for $N=15$. This is corroborated by the mean mixture fraction profiles at $x / D_{1}=6$ [Fig. 27(a)]. Indeed, we can see that the mixture fraction profile is wider for $N=5$ because of the intensity of the ejections. Thus there is an important widening of the outer mixing layer towards the ambient fluid in this case, $\langle f\rangle \neq 0$ until $r / D_{1}=2.4$ for $N=5$ instead of $r / D_{1}=1.3$ for the natural jet. However, this difference between the various $N$ studied occurs only during the transition. When the flow reaches a developed turbulent state, the small-scales appearance leads to an equivalent mixing for the three cases and the mean mixture fraction profiles at $x / D_{1}=10$ are close [Fig. 27(b)]. Therefore, the PDF at the end of the computational domain for the three azimuthal excitations were found to be very similar at the end of the transition (Fig. 28 shows only the case $N=5$ ). As in the axisymmetric forcing case, a marching-type PDF is observed revealing a good homogeneity of the mixing, conversely to 


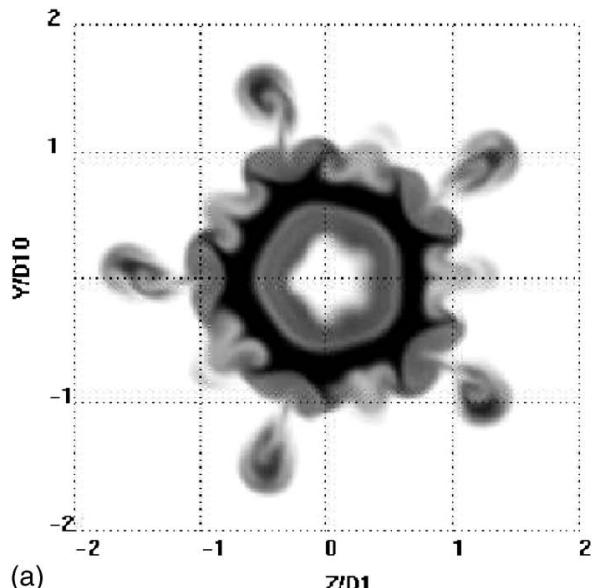

(a)

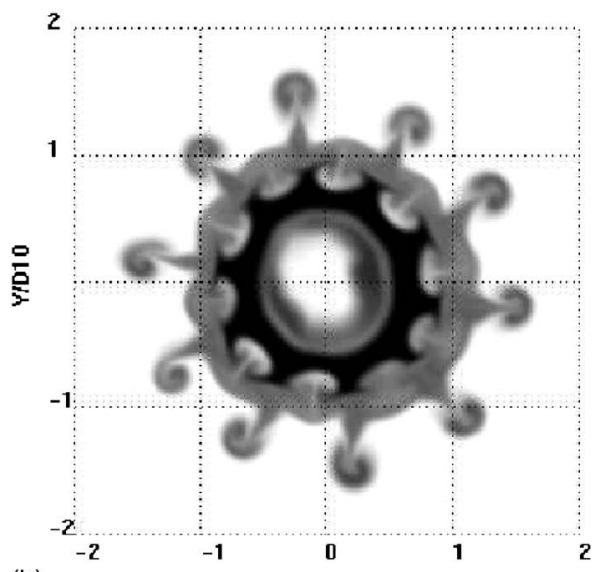

(b)

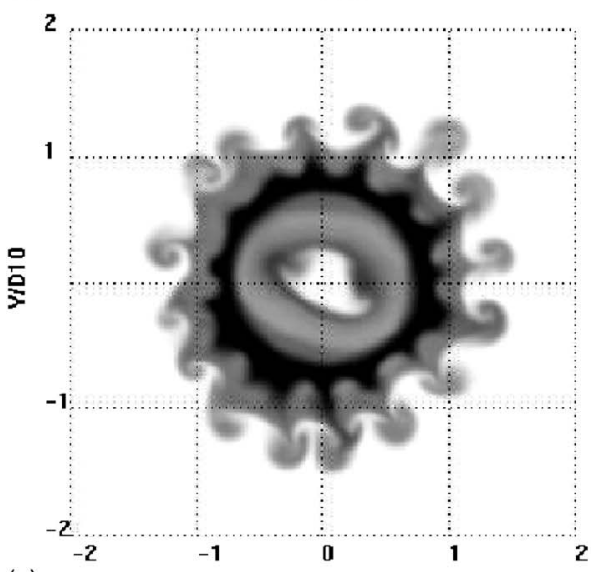

(c)

ZD1

FIG. 26. Instantaneous contours of the mixture fraction in the transverse section located at $x / D_{1}=4.5$ for the three azimuthal excitation cases: (a) $N$ $=5$, (b) $N=10$, and (c) $N=15 . f$ varies from 0 (white) to 1 (black) (enhanced online).

the natural case. The scalar visualizations reveal that no spots of unmixed species can be found at the end of the computational domain.

\section{Mixing efficiency: Comparison between the different jets}

As seen above, the deterministic excitations allow for an improvement of the mixing as compared to the unforced case. In this last part, we then concentrate on global quanti-
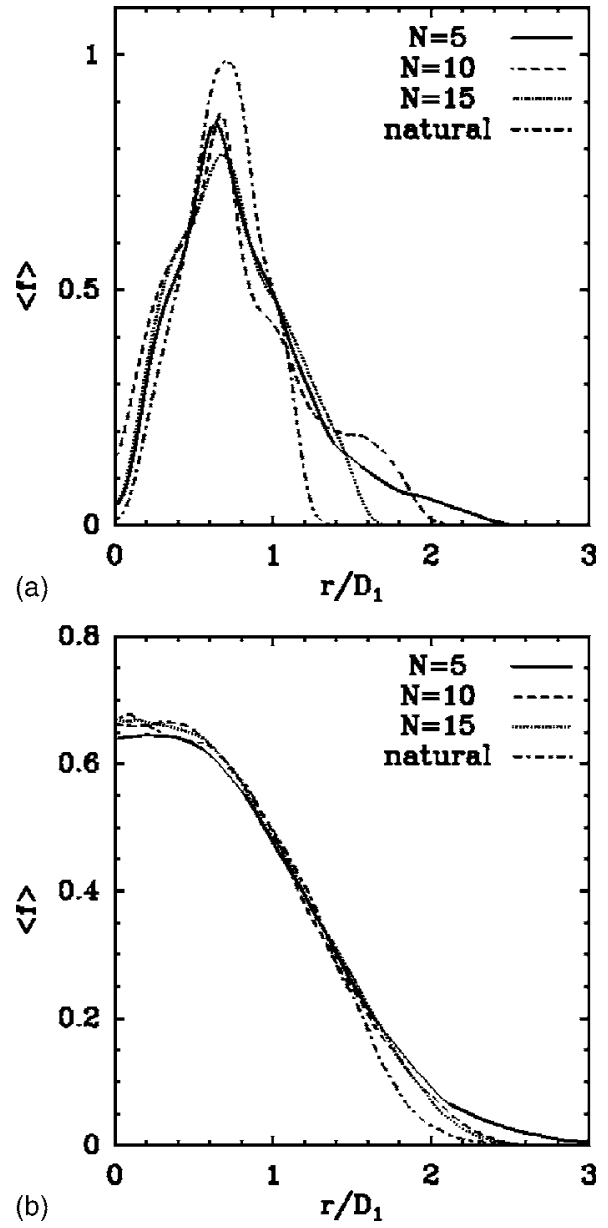

FIG. 27. Mean mixture fraction profiles for the three azimuthal excitations compared to the natural case at $x / D_{1}=6$ (a) and $x / D_{1}=10$ (b).

ties of the jets especially designed to evaluate the mixing efficiency. To demonstrate that the mixing properties are modified by the inflow forcing not only in the near field but also in the far field beyond the end of the outer potential core, the statistics, for the various excitation cases, will be compared at the same distance from this end. We then des-

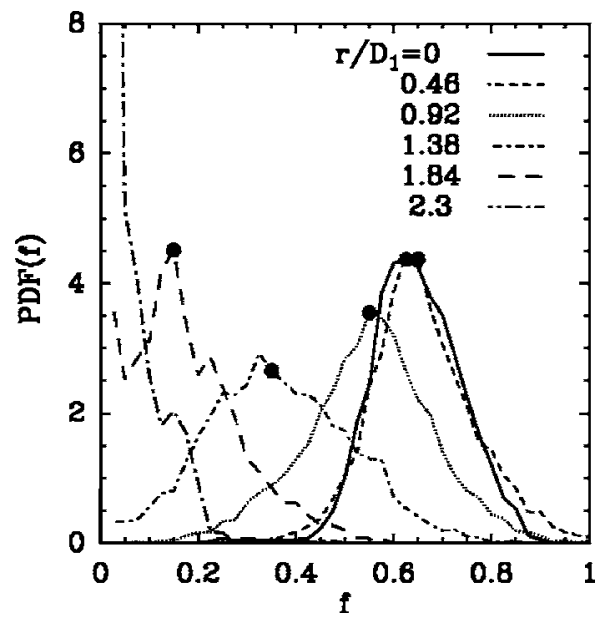

FIG. 28. Variation of the mixture fraction PDF across the mixing layer at $x / D_{1}=10$ for the azimuthal excitation $(N=5)$, the points show the mean value of $f$ for each radial location. 

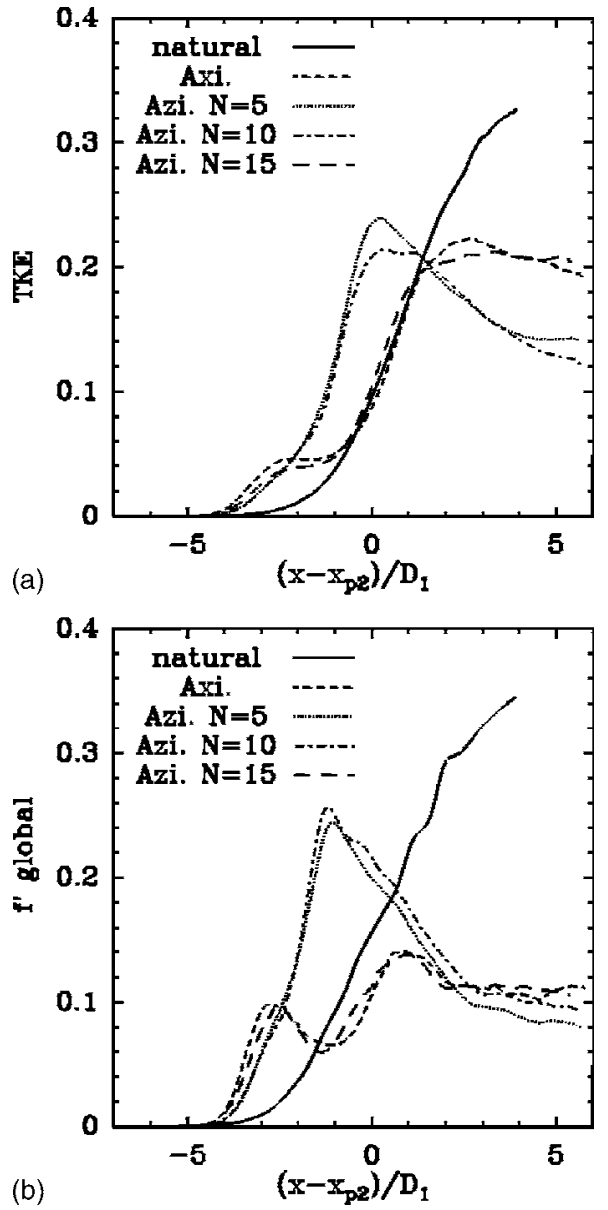

FIG. 29. Downstream evolution of the integral of TKE in section normal to the jet axis (a) and of the integral of $\left\langle f^{\prime 2}\right\rangle$ in the section normal to the jet axis (b) for the different cases: unforced case (natural), axisymmetric excitation (Axi.), and azimuthal excitations (Azi.) for the three wave numbers studied. The downstream evolution is given in function of the outer potential core, $x_{p 2}$.

ignate as $x_{p 2}$ the outer potential core length and plot the downstream evolution of the statistical quantities as a function of $\left(x-x_{p 2}\right) / D_{1}$. Note that $x_{p 2}$ differs from one forcing case to the other. Similarly to previous studies, ${ }^{32,42}$ the outer potential core length is defined as the downstream distance from the inlet at which the mean streamwise velocity starts to differ by more than 5\% from its value at the inlet.

Figures 29(a) and 29(b) show the downstream evolution of the turbulent kinetic energy (TKE), and the mixture fraction rms integrated in sections normal to the jet axis. As previously observed, the azimuthal forcing with small values of the azimuthal wave number $(N=5$ and $N=10)$ allow for an earlier generation of streamwise vortices (before the end of the outer potential core) leading to an early growth of the TKE. These intense streamwise vortices lead to intense radial ejections of the mixture fraction, as previously shown by Fig. 26; this is associated with a rapid growth of the rms of the mixture fraction. It is important to note that the turbulence characteristics are significantly affected by the inflow forcing downstream of the potential core end [i.e., for $(x$ $\left.\left.-x_{p 2}\right) / D_{1}>0\right]$. For the forcing with small values of the azimuthal wave number ( $N=5$ and $N=10)$, the TKE intensity is

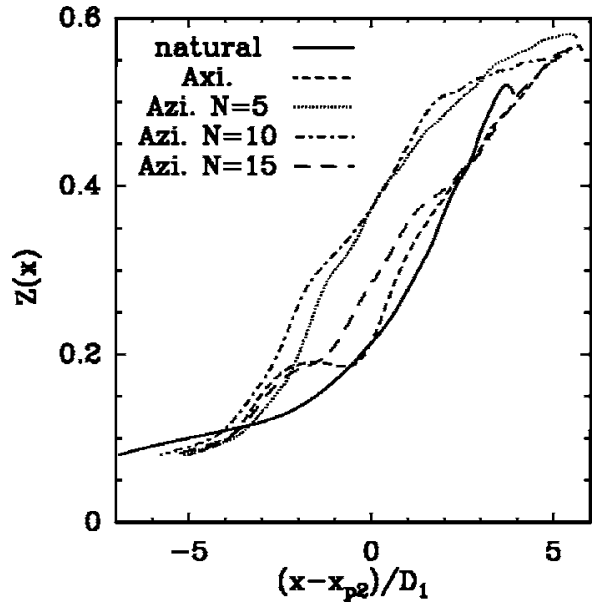

FIG. 30. Downstream evolution of the global mixedness for the different cases: unforced case (natural), axisymmetric excitation (Axi.), and azimuthal excitations (Azi.) for the three wave numbers studied. The downstream evolution is given in function of the outer potential core, $x_{p 2}$.

indeed much smaller than the value observed in the other cases. This indicates a better mixing of momentum with more TKE present in the smallest scales of the flow. Similarly, the mixture fraction rms becomes small for the forced cases showing a good homogeneity of the mixing conversely to the unforced jet. Furthermore, for $\left(x-x_{p 2}\right) / D_{1}>2$, the mixture fraction rms reaches a near constant value in the forced cases with a minimum intensity for $N=5$ a sign of a more efficient mixing.

We next introduce the global mixedness and the intensity of segregation to quantify the mixing improvement due to the jet forcing. The global mixedness $Z(x)$ was introduced by Cetegen and Mohamad. ${ }^{56}$ This quantity is the second moment of the mixture fraction,

$$
Z(x)=\frac{4}{S} \int_{S}\langle f(x, r)\rangle(1-\langle f(x, r)\rangle) r d r d \phi,
$$

where $S$ is the radial section where the integration is performed. $Z(x)$ ranges from 0 for completely unmixed fluids to 1 for complete mixing. Note that this quantity is appropriate when the complete mixing is for bimolecular mixing, i.e., if each species are seeded with the same proportion. Figure 30 shows the downstream evolution of $Z(x)$ for the different cases studied (natural and forced) with the radius of $S$ taken to be equal to $3.5 D_{1}$, allowing the inclusion of all the regions where $\langle f\rangle \neq 0$. For the axisymmetric excitation, the mixing takes place in two distinct stages. First, the mixing is due to the development of intense Kelvin-Helmholtz vortices until the end of the outer potential core. This implies a first rapid growth of $Z(x)$ which reaches a value close to 0.2. Further downstream, $Z(x)$ undergoes a second brutal growth when the streamwise vortices appear (after the outer potential core). Conversely, the mixing grows monotonously during the transition for the azimuthal forcing cases due to the azimuthal deformation of the vortices from the beginning of the jet and the appearance of the streamwise vortices before the end of the outer potential core. Excitations with small values 


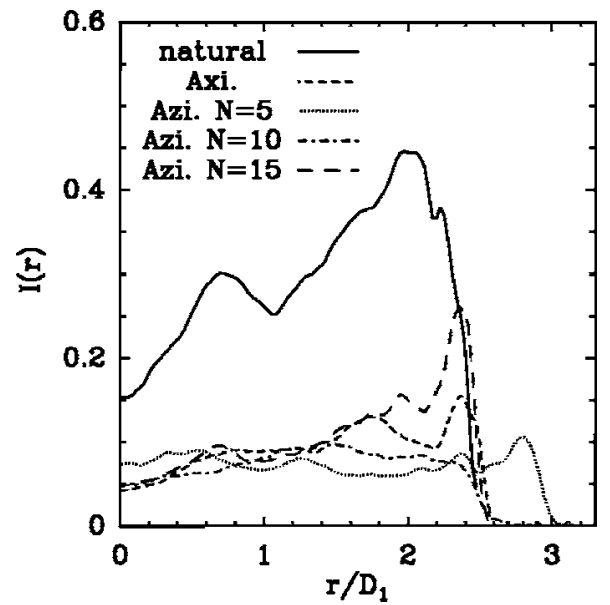

FIG. 31. Radial evolution of the intensity of segregation at $\left(x-x_{p 2}\right) / D_{1}$ $=3.5$ for the different cases: unforced case (natural), axisymmetric excitation (Axi.), and azimuthal excitations (Azi.) for the three wave numbers studied.

of the azimuthal wave number $(N=5$ and $N=10)$ allow an earlier growth of $Z(x)$ because of the faster formation of the streamwise vortices as compared to the other two forced cases $(N=15$ and axisymmetric cases).

To have a better view of the mixing homogeneity after the end of the outer potential core, Fig. 31 shows the intensity of segregation for the different unforced and forced cases at $\left(x-x_{p 2}\right) / D_{1}=3.5$. This quantity, introduced by Danckwerts, ${ }^{57}$ is defined by

$$
I=\frac{\left\langle f^{\prime 2}\right\rangle}{\langle f\rangle(1-\langle f\rangle)} .
$$

It ranges between 0 and 1: $I=1$ when the species remain segregated whereas $I=0$ when a perfectly homogeneous mixing is reached. Figure 31 shows a good homogeneity in the forcing cases conversely to the natural coaxial jet. Within the jet core $\left(r / D_{1}<1.4\right)$, the same mixing efficiency is comparable for all the forced cases. For the axisymmetric excitation and azimuthal excitation with $N=15, I$ becomes larger at the interface between the jet and the ambient fluid; this indicates that the species are stirred but remains weakly segregated in that flow region. Note that, for the $N=5$ excitation, the species is expelled further away from the jet core due the mushroom shape structures associated with the presence of intense longitudinal vortices as observed in Fig. 26.

\section{CONCLUSION}

The goal of the present study was to design inflow forcing procedures aimed at the mixing properties enhancement of coaxial jets at moderate Reynolds number. The forced jets dynamics and mixing properties are compared with the natural jet which was previously studied in details by Balarac et $a l .{ }^{13}$ In the natural jet, it is observed that the transition process begins with the development of Kelvin-Helmholtz vortices on the outer and inner shear layers. There is a domination of the outer Kelvin-Helmholtz vortices which impose their frequency to the inner ones. This stage allows an engulfment of species originally seeded in the outer jet through the inner and outer shear layers. Further downstream, counter-rotating pairs of streamwise vortices appear allowing the ejections of these species. They are characterized by mushroom-type structures. These ejections play an important role in the mixing process of the jet. However, at the end of the transition, the mixing in the natural coaxial jet is not homogeneous and spots of unmixed fluid remain. We have then designed deterministic excitations to allow a better mixing. Since the vortices of the outer shear layer were found to play a dominant role in the natural jet, the excitations were applied only in this outer region. Moreover, excitations with moderate amplitude were considered because of their greater interest for potential industrial applications.

Two different types of forcing were investigated. The first forcing consists of a purely axisymmetric excitation at the most unstable frequency of the outer shear layer allowing the faster development of Kelvin-Helmholtz instability of both outer and inner layers. Due to the nature of the forcing, these were found to be very axisymmetric. These intense vortices allow the engulfment of the outer stream from the beginning of the jet. Furthermore, a faster transition was observed since streamwise vortices are formed earlier and so the ejection phenomenon of the outer species is faster as compared to the unforced case. The mean flow is also strongly influenced by this forcing with a diminution of the outer and inner potential core length. Finally, the mixing is found to be more homogeneous with the disappearance of spots of unmixed species at the end of the computational domain.

The second type of forcing consists of a combination of an axisymmetric forcing and an azimuthal excitation with an azimuthal wave number $N$. This mimics $N$ microjets placed circumferentially around the outer nozzle and ejecting periodically. Similarly to the purely axisymmetric case, the excitation is applied at the outer shear layer most amplified frequency. This triggers a rapid development of the outer and inner Kelvin-Helmholtz vortices implying an efficient engulfment of the outer species. The azimuthal disturbance yields an azimuthal deformation of the outer KelvinHelmholtz vortices leading to the early formation of counterrotating streamwise vortices. They are more intense during the transition for small values of the azimuthal wave number. Contrary to the Kelvin-Helmholtz instabilities, the outer azimuthal instabilities do not disturb the inner shear layer, and the inner Kelvin-Helmholtz vortices keep their axisymmetry. The outer streamwise vortices allow us to generate intense ejection of the species seeded in the outer jet. With these azimuthal excitations, the mixedness is found to be homogeneous without spots of unmixed fluids.

Finally, we quantify the mixedness efficiency associated with the various types of forcing through the global mixedness and the intensity of segregation. These quantities reveal the good mixing homogeneity at the end of the computational domain in all forced cases compared with the natural case. The global mixedness allows also to show that the purely axisymmetric excitation leads to a mixing development in two parts: due to the Kelvin-Helmholtz vortices, and later, due to the streamwise vortices. There is moreover an 
early growth of the global mixedness for the excited cases with small values of the azimuthal wave number $N$ because of the early formation of streamwise vortices.

\section{ACKNOWLEDGMENT}

Part of the computations were carried out at the Institut du Développement et des Ressources en Informatique Scientifique (IDRIS, France).

${ }^{1}$ F. H. Champagne and I. J. Wygnanski, "An experimental investigation of coaxial turbulent jets," Int. J. Heat Mass Transfer 14, 1445 (1971).

${ }^{2}$ S. C. Crow and F. H. Champagne, "Orderly structure in jet turbulence," J. Fluid Mech. 48, 547 (1971).

${ }^{3}$ E. Villermaux and H. Rehab, "Mixing in coaxial jets," J. Fluid Mech. 425, 161 (2000)

${ }^{4}$ N. W. M. Ko and A. S. H. Kwan, "The initial region of subsonic coaxial jets," J. Fluid Mech. 73, 305 (1976).

${ }^{5}$ A. S. H. Kwan and N. W. M. Ko, "The initial region of subsonic coaxial jets. Part 2," J. Fluid Mech. 82, 273 (1977).

${ }^{6}$ N. W. M. Ko and H. Au, "Initial region of subsonic coaxial jets of high mean-velocity ratio," J. Fluid Mech. 103, 335 (1981).

${ }^{7}$ W. J. A. Dahm, C. E. Frieler, and G. Tryggvason, "Vortex structure and dynamics in the near field of a coaxial jet," J. Fluid Mech. 241, 371 (1992).

${ }^{8}$ G. Buresti, P. Petagna, and A. Talamelli, "Experimental investigation on the turbulent near-field of coaxial jets," Exp. Therm. Fluid Sci. 17, 18 (1998).

${ }^{9}$ R. B. Wicker and J. K. Eaton, "Near field of a coaxial jet with and without axial excitation," AIAA J. 32, 542 (1994).

${ }^{10} \mathrm{G}$. Balarac and O. Métais, "Coherent vortices in coaxial jets," in Advances in Turbulence X, edited by H. I. Anderson and P.-Å. Krogstad (CIMNE, Barcelona, 2004).

${ }^{11}$ L. P. Bernal and A. Roshko, "Streamwise vortex structure in plane mixing layers," J. Fluid Mech. 170, 449 (1986).

${ }^{12} \mathrm{D}$. Liepmann and M. Gharib, "The role of the streamwise vorticity in the near-field entrainment of a round jet," J. Fluid Mech. 245, 643 (1992).

${ }^{13}$ G. Balarac, M. Si-Ameur, M. Lesieur, and O. Métais, "Direct numerical simulations of high velocity ratio coaxial jets: Mixing properties and influence of the upstream conditions," J. Turbul. 8, 1 (2007).

${ }^{14} \mathrm{H}$. Rehab, E. Villermaux, and E. J. Hopfinger, "Flow regimes of largevelocity-ratio coaxial jets," J. Fluid Mech. 345, 357 (1997).

${ }^{15}$ C. B. da Silva, G. Balarac, and O. Métais, "Transition in high velocity ratio coaxial jets analysed from direct numerical simulations," J. Turbul. 4, 1 (2003).

${ }^{16}$ N. Kurimoto, Y. Suzuki, and N. Kasagi, "Active control of lifted diffusion flames with arrayed micro actuators," Exp. Fluids 39, 995 (2005).

${ }^{17}$ N. Kurimoto, Y. Suzuki, and N. Horiuchi, "Active control of coaxial jet mixing and combustion with arrayed micro actuators," 5th World Conference on Experimental Heat Transfer, Fluid Mechanics, and Thermodynamics, Thessaloniki, September 2001, pp. 511-516.

${ }^{18}$ K. Angele, N. Kurimoto, Y. Suzuki, and N. Kasagi, "Evolution of the streamwise vortices in coaxial jet controlled with micro flap actuators," J. Turbul. 7, 1 (2006).

${ }^{19}$ E. J. Gutmark and F. F. Grinstein, "Flow control with noncircular jets," Annu. Rev. Fluid Mech. 31, 239 (1999).

${ }^{20}$ K. B. M. Q. Zaman, "Spreading characteristics of compressible jets from nozzles of various geometries," J. Fluid Mech. 383, 197 (1999).

${ }^{21}$ N. H. Saiyed and J. E. Bridges, "Tabs and mixers for reducing low bypass ratio jet noise," AIAA Pap. No. 99-1986 (1999).

${ }^{22}$ V. H. Arakeri, A. Krothappalli, V. Siddavaram, M. B. Alkislar, and L. M. Lourenco, "On the use of microjets to suppress turbulence in a Mach 0.9 axisymmetric jet," J. Fluid Mech. 490, 75 (2003).

${ }^{23}$ C. B. da Silva and O. Métais, "Vortex control of bifurcating jets: a numerical study," Phys. Fluids 14, 3798 (2002).

${ }^{24}$ W. C. Reynolds, D. E. Parekh, P. J. D. Juvet, and M. J. D. Lee, "Bifurcating and blooming jets," Annu. Rev. Fluid Mech. 35, 295 (2003).

${ }^{25}$ I. Danaila and B. J. Boersma, "Direct numerical simulation of bifurcating jets," Phys. Fluids 12, 1255 (2000).

${ }^{26}$ J. B. Freund and P. Moin, "Jet mixing enhancement by high-amplitude fluidic actuation," AIAA J. 38, 1863 (2000).

${ }^{27}$ A. Hilgers, "Control and optimization of turbulent jet mixing," in Annual
Research Briefs (Center of Turbulence Research, Stanford University, Stanford, 2000).

${ }^{28}$ A. Hilgers and B. J. Boersma, "Optimization of turbulent jet mixing," Fluid Dyn. Res. 29, 345 (2001).

${ }^{29}$ D. J. Bodony and S. K. Lele, "Review of the current status of jet noise predictions using large-eddy simulation," AIAA Pap. No. 2006-0468 (2006).

${ }^{30}$ P. J. Morris, L. N. Long, T. E. Scheidegger and S. Boluriaan, "Simulations of supersonic jet noise," Inter. J. Aeroacoustics 1, 17 (2002).

${ }^{31}$ C. Bogey and C. Bailly, "LES of a high Reynolds, high subsonic jet: Effects of the inflow conditions on flow and noise," AIAA Pap. No. 20033170 (2003).

${ }^{32} \mathrm{C}$. Bogey and C. Bailly, "Effects of inflow conditions and forcing on subsonic jet flows and noise," AIAA J. 43, 1000 (2005).

${ }^{33}$ N. Andersson, L.-E. Eriksson and L. Davidson, "Effects of inflow conditions and subgrid model on LES for turbulent jets," AIAA Pap. No. 20052925 (2005).

${ }^{34}$ B. D. Ritchie, D. R. Mujumdar, and J. M. Seitzman, "Mixing in coaxial jets using synthetic jet actuators," AIAA Pap. No. 2000-0404 (2000).

${ }^{35}$ C. Canuto, M. Y. Hussaini, A. Quarteroni, and T. A. Zang, Spectral Methods in Fluid Dynamics (Springer, New-York, 1987).

${ }^{36}$ S. K. Lele, "Compact finite difference schemes with spectral-like resolution," J. Comput. Phys. 103, 15 (1992).

${ }^{37}$ J. H. Williamson, "Low-storage Runge-Kutta schemes," J. Comput. Phys. 35, 48 (1980).

${ }^{38}$ I. Orlanski, "A simple boundary condition for unbounded hyperbolic flows," J. Comput. Phys. 21, 251 (1976).

${ }^{39}$ M. A. Gonze, "Simulation numérique des sillages en transition à la turbulence," Ph.D. thesis, Institut National Polytechnique de Grenoble, 1993.

${ }^{40}$ C. Hirsch, Numerical Computation of Internal and External Flows, Vol. 2 (Wiley, New York, 2002).

${ }^{41}$ C. B. da Silva and O. Métais, "On the influence of coherent structures upon interscale interactions in turbulent plane jets," J. Fluid Mech. 473, 103 (2002)

${ }^{42} \mathrm{G}$. Balarac and O. Métais, "The near field of coaxial jets: A numerical study," Phys. Fluids 17, 065102 (2005).

${ }^{43}$ M. Maidi, M. Lesieur, and O. Métais, "Vortex control in large-eddy simulations of compressible round jets," J. Turbul. 7, 1 (2006).

${ }^{44}$ S. E. Widnall, D. B. Bliss, and C. Y. Tsai, "The instability of short waves on a vortex ring," J. Fluid Mech. 66, 35 (1974).

${ }^{45}$ T. Maxworthy, "Some experimental studies of vortex rings," J. Fluid Mech. 81, 465 (1977).

${ }^{46}$ P. G. Saffman, "Number of waves on unstable vortex rings," J. Fluid Mech. 84, 625 (1978).

${ }^{47}$ J. Choi, A. M. Annaswamy, O. Egungwu, and F. S. Alvi, "Active noise control of supersonic impinging jet using pulsed microjets," AIAA Pap. No. 2005-798 (2005)

${ }^{48}$ M. K. Ibrahim, R. Kunimura and Y. Nakamura, "Spreading enhancement of axisymmetric supersonic jet by the use of micro jets," AIAA Pap. No. 2001-31351 (2001).

${ }^{49}$ M. Soteriou, R. Reba and T. Maeder, "Numerical study of the impact of streamwise vorticity on jet noise," AIAA Pap. No. 2002-2480 (2002).

${ }^{50} \mathrm{C}$. Bogey, C. Bailly and D. Juvé, "Noise investigation of a high subsonic, moderate Reynolds number using a compressible large eddy simulation," Theor. Comput. Fluid Dyn. 16, 273 (2003).

${ }^{51}$ J. C. R. Hunt, A. A. Wray, and P. Moin, "Eddies, stream, and convergence zones in turbulent flows," in Annual Research Briefs (Center of Turbulence Research, Stanford University, Stanford, 1988).

${ }^{52}$ Y. Dubief and F. Delcayre, "On coherent vortex identification in turbulence," J. Turbul. 1, 11 (2000).

${ }^{53}$ M. Lesieur, O. Métais, and P. Comte, Large-Eddy Simulations of Turbulence (Cambridge University Press, Cambridge, 2005).

${ }^{54}$ S. A. Stanley, S. Sarkar, and J. P. Mellado, "A study of the flow-field evolution and the mixing in a plane turbulent jet using direct numerical simulation," J. Fluid Mech. 450, 377 (2002).

${ }^{55}$ L. M. Pickett and J. B. Ghandhi, "Passive scalar mixing in a planar shear layer with laminar and turbulent inlet conditions," Phys. Fluids 14, 985 (2002).

${ }^{56}$ B. M. Cetegen and N. Mohamad, "Experiments on liquid mixing and reaction in a vortex," J. Fluid Mech. 249, 391 (1993).

${ }^{57} \mathrm{P}$. V. Danckwerts, "The definition and measurement of some characteristics of mixture," Appl. Sci. Res., Sect. A 3, 279 (1952). 